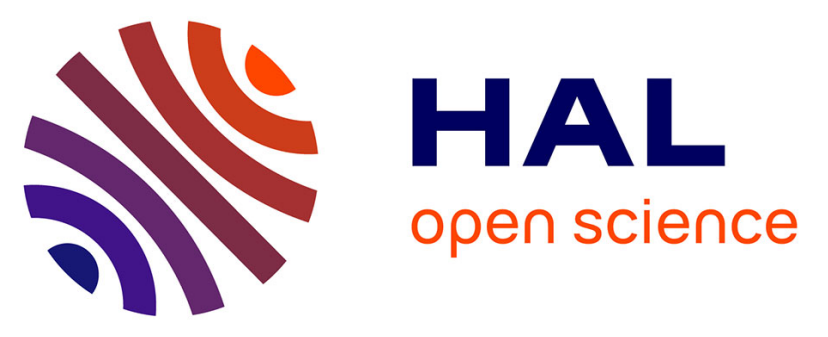

\title{
Synthesis and in vitro evaluation of 4-trichloromethylpyrrolo[1,2-a]quinoxalines as new antiplasmodial agents
}

Nicolas Primas, Peggy Suzanne, Pierre Verhaeghe, Sébastien Hutter, Charline Kieffer, Michèle Laget, Anita Cohen, Julie Broggi, Jean-Charles Lancelot, Aurélien Lesnard, et al.

\section{To cite this version:}

Nicolas Primas, Peggy Suzanne, Pierre Verhaeghe, Sébastien Hutter, Charline Kieffer, et al.. Synthesis and in vitro evaluation of 4-trichloromethylpyrrolo[1,2-a]quinoxalines as new antiplasmodial agents. European Journal of Medicinal Chemistry, 2014, 83, pp.26-35. 10.1016/j.ejmech.2014.06.014 . hal01417058

\author{
HAL Id: hal-01417058 \\ https://hal.science/hal-01417058
}

Submitted on 15 Dec 2016

HAL is a multi-disciplinary open access archive for the deposit and dissemination of scientific research documents, whether they are published or not. The documents may come from teaching and research institutions in France or abroad, or from public or private research centers.
L'archive ouverte pluridisciplinaire HAL, est destinée au dépôt et à la diffusion de documents scientifiques de niveau recherche, publiés ou non, émanant des établissements d'enseignement et de recherche français ou étrangers, des laboratoires publics ou privés. 


\title{
Synthesis and in vitro evaluation of 4-trichloromethylpyrrolo[1,2- a]quinoxalines as new antiplasmodial agents.
}

Nicolas Primas ${ }^{1}$, Peggy Suzanne ${ }^{2}$, Pierre Verhaeghe ${ }^{3}$, Sébastien Hutter ${ }^{4}$, Charline Kieffer ${ }^{1}$, Michèle Laget ${ }^{4}$, Anita Cohen ${ }^{4}$, Julie Broggi ${ }^{1}$, Jean-Charles Lancelot ${ }^{2}$, Aurélien Lesnard ${ }^{2}$, Patrick Dallemagne ${ }^{2}$, Pascal Rathelot ${ }^{1}$, Sylvain Rault ${ }^{2}$, Patrice Vanelle ${ }^{1, *}$ and Nadine Azas ${ }^{4, *}$

${ }^{1}$ Aix-Marseille Université, CNRS, ICR UMR 7273, Laboratoire de Pharmaco-Chimie Radicalaire,Faculté de Pharmacie, 27 Boulevard Jean Moulin - CS30064, 13385 Marseille cedex 05, France.

${ }^{2}$ Université de Caen Basse Normandie, Faculté des Sciences Pharmaceutiques, Centre d'Etudes et de Recherche sur le Médicament de Normandie (CERMN) - EA 4258, FR CNRS INC3M, Boulevard Becquerel, 14032 Caen, France.

${ }^{3}$ Université Paul Sabatier, Faculté des Sciences Pharmaceutiques - CNRS UPR 8241, Laboratoire de Chimie de Coordination, 205 Route de Narbonne, 31077 Toulouse cedex 04, France.

${ }^{4}$ Aix-Marseille Université, UMR MD 3, Infections Parasitaires, Transmission et Thérapeutique, Faculté de Pharmacie, 27 Boulevard Jean Moulin - CS30064, 13385 Marseille cedex 05, France.

*Corresponding authors: Patrice Vanelle, Tel.: +33491835580; Fax: +33491794677; email: patrice.vanelle@univ-amu.fr. Nadine Azas, Tel.: +33491835564; email: nadine.azas@univamu.fr.

\begin{abstract}
:
Thanks to a preliminary in vitro screening of several $\mathrm{CCl}_{3}$-substituted-nitrogen containing heterocycles belonging to our chemical library, the 2-trichloromethylquinoxaline scaffold appeared to be of potential interest for developing new antiplasmodial agents. Then, combining these experimental results to the antimalarial properties reported for variouspyrrolo[1,2-a]quinoxaline derivatives, an original series of fifteen 7-substituted-4trichoromethylpyrrolo[1,2-a]quinoxalines was synthesized in a 4 to 5 reaction steps pathway. All molecules were evaluated in vitro toward both their antiplasmodial activity on the K1 multi-resistant $P$. falciparum strain and their cytotoxicity on the HepG2 human cell line.
\end{abstract}


Thus, 3 hit molecules were identified, displaying $\mathrm{IC}_{50}$ values in the micromolar range and low cytotoxicity values, reaching good selectivity indexes, in comparison with the reference drugs chloroquine and doxycycline. Structure-activity relationship studies showed that the pyrrolo[1,2-a]quinoxaline scaffold can support selective antiplasmodial activity when substituted at position 4 by a $\mathrm{CCl}_{3}$ group. However, substitution at position 7 of the same scaffold is neither beneficial for cytotoxicity nor favorable for the solubility in the biological media.

Keywords:Plasmodium falciparum;Pyrrolo[1,2-a]quinoxaline; Trichloromethyl goup;In vitro antiplasmodial activity; In vitro HepG2 cytotoxicity, SARs.

\section{Introduction}

Nowadays, malaria still remains one of the most prevailing and lethal infectious disease worldwide. The protozoa parasite responsible for this infection belongs to the Plasmodiumgenus and is transmitted to humans by the bite of the infectedAnopheles mosquito female. Among the fivePlasmodium species responsible for malaria, the most lethal is $P$. falciparum, causing cerebral malaria. According to the WHO, in 2012, malaria affected about 207 million people in the inter-tropical area and caused an estimated 627,000 deaths [1]. Artemisinin-based combination therapies (ACTs) are recommended by the WHO as the firstline treatment for treating malaria caused by $P$. falciparum. Unfortunately, parasite resistance to artemisinin derivatives has now been detected in 4 countries of the Greater Mekong subregion: Cambodia, Myanmar, Thailand and Vietnam [2-6]. This emerging resistance of $P$. falciparum strains is a serious problem for the containment and the treatment of this disease as it could lead to a resurgence of more virulent levels of malaria, unless new chemical entities,displaying novel mechanisms of action, be rapidly found [7-10].

Aiming at synthesizing original molecules displaying antiparasitic properties [11-15], our research group previously reported promising results in 4-substituted-2trichloromethylquinazoline series, showing that the trichloromethyl group was crucial for the in vitro activity against Plasmodium falciparum [16-20]. These results prompted us to investigate wider the anti-infectious potential of various aromatic nitrogen-containing 
heterocyclic scaffolds bearing a trichloromethyl group, in a view to identify new antiplasmodial candidates.

\section{Results and discussion}

\subsection{Preliminary antiplasmodial screening of various trichloromethylated heterocyclic scaffolds}

An antiplasmodial screening was first made from a series of closely related compounds corresponding to aromatic 6-membered bicyclic nitrogen-containing heterocycles bearing a $\mathrm{CCl}_{3}$ group. These compounds were selected in our internal chemical library, part of the French National Chemical Library(Chimiothèque Nationale). Thus, quinoline (series 1), isoquinoline (series 2), quinazoline (series 3 and 4) and quinoxaline (series 5)derivatives (Figure 1) were evaluated in vitro toward both their antiplasmodial activity against the K1 multi-resistant $P$. falciparum strain (determination of the $\mathrm{IC}_{50}=$ inhibitory concentration $50 \%$ ) and their cytotoxicity (determination of the $\mathrm{CC}_{50}=$ cytotoxic concentration $50 \%$ ) on the HepG2 human cell line.

\section{<Insert Figure 1.>}

Molecules 1a, 2a, 4a and 5a were prepared from their corresponding methylated precursors (1b, $\mathbf{2 b}$ and $\mathbf{5 b}$, purchased from Sigma-Aldrich) by a microwave-assisted chlorination reaction using $\mathrm{PCl}_{5}$ and $\mathrm{POCl}_{3}[21,22]$. Molecule $\mathbf{4 b}$ was prepared as described by Liu and co-workers by reacting acetamidine hydrochloride with 2-bromobenzylamine in the presence of $\mathrm{CuBr}$ as catalyst [23].Compound 3awas obtained by using an alternative route which was previously developed in our lab [24,25]. Thus, 4-chloroquinazoline was reacted with bromotrichloromethane in the presence of tetrakis(dimethylamino)ethylene (TDAE) instead of using hazardous chlorine gas, as described in the literature [26]. Then, all these molecules were tested in vitro and compared with two commercial reference-drugs: chloroquine and doxycycline. For all tested compounds, the corresponding selectivity indexes (SI) were calculated. The results are presented in Table 1.

\section{<Insert Table 1.>}

Among all tested compounds, only the quinoline, isoquinoline or quinoxaline derivatives bearing a $\mathrm{CCl}_{3}$ group showed some potential in vitro antiplasmodial activity (respectively 
compounds 1a, 2a and 5a), in comparison with chloroquine or doxycycline. The quinazoline derivatives including a $\mathrm{CCl}_{3}$ group at position 2 or 4 (compounds $\mathbf{4 a}$ and 3a) did not display significant activity. These last results indicated that the $\mathrm{CCl}_{3}$ group was not sufficient alone for providing antiplasmodial activity in quinazoline series and suggested that the position of the $\mathrm{CCl}_{3}$ group on the quinazoline ring could also play a key-role toward biological activity. Moreover, when comparing the activities of the $\mathrm{CCl}_{3}$ containing compounds to their $\mathrm{CH}_{3}$ analogs, in all tested series, it clearly appeared that the $\mathrm{CCl}_{3}$ group was involved in the antiplasmodial activity, in accordance with the experimental results which we previously obtained in 4-substituted quinazoline series [16-19].

Although displaying antiplasmodial activity, quinoline 1a and isoquinoline $\mathbf{2 a}$ did not appear to be the most potent compounds because of their rather high $\mathrm{IC}_{50}$ values (respectively 8.5 and $8 \mu \mathrm{M})$, in comparison with quinoxaline $5 \mathbf{a}\left(\mathrm{IC}_{50}=1.5 \mu \mathrm{M}\right)$ and reference-drugs chloroquine $\left(\mathrm{IC}_{50}=0.6 \mu \mathrm{M}\right)$ and doxycycline $\left(\mathrm{IC}_{50}=6.0 \mu \mathrm{M}\right)$. Nevertheless, 5a showed a significant in vitro cytotoxicity $\left(\mathrm{CC}_{50}=3.1 \mu \mathrm{M}\right)$ in comparison with doxorubicine $\left(\mathrm{CC}_{50}=0.2\right.$ $\mu \mathrm{M}$ ), making this compound poorly selective and unsuitable for further development. In order to validate the SARs of $\mathbf{5 a}$, in addition to its methylated precursor $\mathbf{5 b}$, three closely related analogs (5c, 5d and 5e) were also tested (Figure 2). Compound 5c was prepared by a chlorination reaction of $\mathbf{5 b}$ using $\mathrm{PCl}_{5}$ and $\mathrm{POCl}_{3}$, under milder conditions than those used to prepare 5a, while compounds $\mathbf{5 d}$ and $\mathbf{5 e}$ were purchased from Aldrich.

\section{<Insert Figure 2.>}

The comparison of the results noted for the tested quinoxaline derivatives 5a-e indicated that the quinoxaline ring, itself, did not show any antiplasmodial potential $\left(\mathrm{IC}_{50}\right.$ of $\mathbf{5 e}>50$ $\mu \mathrm{M})$ and that the $\mathrm{CCl}_{3}$ group was the only one that could confer antiplasmodial potential to the studied series, when located at position 2 of the quinoxaline ring (Table 1).

Then, trying to reach a new 2-trichloromethylquinoxaline derivative presenting an improved antiplasmodial profile, we synthesized compound $\mathbf{5 f}$ in a view to compare its biological profile to the one of its antiplasmodial quinazoline position isomer which we previously reported [16](Figure 3). 5f was prepared from its corresponding methylated precursor which was synthesized according to the literature [27] by a microwave-assisted chlorination reaction using $\mathrm{PCl}_{5}$ and $\mathrm{POCl}_{3}$. 


\section{<Insert Figure 3. $>$}

Indeed, quinoxaline $\mathbf{5 f}$ appeared to be much more selective than $\mathbf{5 a}(\mathrm{SI}=17.5$ versus 2.1, respectively), presenting a quite low antiplasmodial $\mathrm{IC}_{50}$ value $(0.2 \mu \mathrm{M})$, better than the one of its quinazoline position isomer $\left(\mathrm{IC}_{50}=8 \mu \mathrm{M}\right)$. However, quinoxaline $\mathbf{5 f}$ remained too cytotoxic $\left(\mathrm{CC}_{50}=3.5 \mu \mathrm{M}\right)$ for allowing further investigations.

Such interesting result noted with compound $\mathbf{5 f}$ prompted us to carry on the investigation of the antiplasmodial potential of the 2-trichloromethylquinoxaline scaffold, especially when considering that various pyrrolo[1,2-a]quinoxaline derivatives (Figure 4) had been reported in the literature as quite promising antimalarial compounds [28-31].

\section{<Insert Figure 4.>}

Taking advantage of the synthetic know-how of our research teams in the fields of both trichloromethylated heterocycles [15-21] and pyrrolo[1,2-a]quinoxalines [32] preparation, we studied the synthesis and antiplasmodial properties of a series of original molecules, based on the quinoxaline ring. These molecules, corresponding to 4-(trichloromethyl)pyrrolo[1,2a]quinoxaline derivatives, were a combination between the pyrrolo[1,2-a]quinoxaline scaffold and the trichloromethyl group (Figure 5), aiming at identifying new antiplasmodial quinoxalines with improved biological profile, being less cytotoxic than $\mathbf{5 f}$.

\section{$<$ Insert Figure 5. $>$}

\subsection{Synthesis of the 4-(trichloromethyl)pyrrolo[1,2-a]quinoxaline series}

The 7-substituted-4-(trichloromethyl)pyrrolo[1,2-a]quinoxalines derivatives were synthesized from various commercially available 4-substituted-2-nitroanilines 6a-i (Scheme 1). The Clauson-Kaas reaction between anilines and 2,5-dimethoxytetrahydrofuran (2,5DMTHF), in refluxing glacial $\mathrm{AcOH}$, provided the pyrrolic derivatives7a-i in 63-86\% yields. The nitro group was then reduced, either by $\mathrm{BiCl}_{3} / \mathrm{NaBH}_{4}$ or tin (II) chloride in refluxing ethanol, affording the expected 1-(2-amino-4-substituted-phenyl)pyrroles8a-i in moderate to good yields(47-90\%). Subsequent acylation of the aniline moiety with trichloroacetyl chloride furnished the corresponding trichloroacetamides 9a-i in $46-88 \%$ yields. These final intermediates were cyclized under Bischler-Napieralski conditions, leading to the expected 7substituted-4-(trichloromethyl)pyrrolo[1,2-a]quinoxalines 10a-i in 20-69\% yields. The 
carboxylic acid derivative $\mathbf{1 0 j}$ was prepared in $44 \%$ yield from methylic ester 10i by an acidic hydrolysis in concentrated $\mathrm{HCl}$. Amidoxime10k was obtained in $74 \%$ yield by reacting nitrile derivative 10h with hydroxylamine hydrochloride in a refluxing $\mathrm{EtOH} / \mathrm{H}_{2} \mathrm{O}$ mixture. Compounds 11a, 11f and 11h were obtained, respectively, by reducing the trichloromethyl group of 10a, 10f and 10h with iron in refluxing glacial AcOH. Compound 12a [33] was prepared by trifluoroacetylation of aniline $\mathbf{8 a}$ in the presence of trifluoroacetic acid, trichloroacetonitrile and triphenylphosphine [34] followed by a Bischler-Napieralski cyclisation.

\section{<Insert Scheme1.>}

\subsection{Biological evaluation of the 7-substituted-4-(trichloromethyl)pyrrolo[1,2- a]quinoxaline derivatives}

Thus, a series of fifteen 4-trichloromethylpyrrolo[1,2-a]quinoxaline derivatives was then tested in vitro for both its in vitro cytotoxicity (human HepG2 cell line) and its antiplasmodial activity (K1 P. falciparummulti-resistant strain). The results (Table 2) were compared to the ones obtained with chloroquine and doxycycline, chosen as antimalarial reference-drugs and doxorubicin, chosen as a reference cytotoxic agent.

\section{<Insert Table2.>}

Among the eleven tested compounds bearing a trichloromethyl group, seven exerted a significant antiplasmodial activity in comparison with the 2 antimalarial drug references, presenting $\mathrm{IC}_{50}$ values in the micromolar range $(1.2$ to $2.4 \mu \mathrm{M})$. Out of these seven hit compounds, four molecules displayed a limited cytotoxicity toward the HepG2 human cell line, with $\mathrm{CC}_{50}$ values ranging from $>15$ to $53 \mu \mathrm{M}$, in comparison with reference-drug doxorubucine $\left(\mathrm{CC}_{50}=0.2 \mu \mathrm{M}\right)$. Thus, three of these molecules reached a SI superior to 10 $\mu \mathrm{M}$ : 10a, 10f and 10h. Indeed, hit compound 10a demonstrated the same antiplasmodial activity as starting compound $\mathbf{5 a}\left(\mathrm{IC}_{50}=1.5 \mu \mathrm{M}\right)$ but showed a greatly improved cytotoxicity $\left(\mathrm{CC}_{50}=41.2 \mu \mathrm{M}\right)$, making its selectivity $(\mathrm{SI}=27.5)$ intermediate between the one of chloroquine $(\mathrm{SI}=50)$ and the one of doxycycline $(\mathrm{SI}=3.3)$. It could then be concluded that the attachment of a pyrrole moiety to the quinoxaline ring clearly improved the antiplasmodial profile of the 2-trichloromethylquinoxaline scaffold. Due to a lack of solubility in the biological media, compounds $\mathbf{1 0 d}$ and $\mathbf{1 0} \mathbf{i}$ could not be tested. 
Concerning the influence of the nature of the substituents at position 7 of the pyrrolo[1,2a]quinoxaline scaffold, it appeared that halogen substituents have a negative influence on the cytotoxicity profile without improving the antiplasmodial activity. Moreover, the carboxylic acid derivative $\mathbf{1 0 j}$ was not active. Globally, no substituent at position 7 of the chemical scaffold was required for providing antiplasmodial activity; some substituents (bromine, iodine, methyl carboxylate or hydroxyamidine) could even play a negative role toward either cytotoxicity or solubility in the biological media.

In order to study the influence of the trichloromethyl group at position 4 of the pyrroloquinoxaline scaffold, 4-methylsubstituted analogs 11a, 11f and 11h of respective hit compounds 10a, 10f and 10h were prepared and evaluated. The results clearly showed that these 4-methylpyrrolo[1,2-a]quinoxaline analogs do not show any activity against $P$. falciparum. Moreover, the 4- $\mathrm{CF}_{3}$ substituted analog 12a of hit compound 10a was not active neither, demonstrating that the $\mathrm{CCl}_{3}$ group is very specifically required for providing antiplasmodial activity in 4-trichloromethylpyrrolo[1,2-a]quinoxaline series.

\section{Conclusion}

From a preliminary screening, highlighting that the 2-trichloromethylquinoxaline scaffold displayed antiplasmodial potential, a new series of 7-substituted-4-trichloromethylpyrrolo[1,2-a]quinoxaline derivatives was synthesized in four to five reaction steps. Thus, three hit compounds were identified, presenting in vitro $\mathrm{IC}_{50}$ values in the micromolar range $(1.5 \leq \mathrm{K} 1$ P. falciparum $\left.\mathrm{IC}_{50} \leq 2.4 \mu \mathrm{M}\right)$, good cytotoxity profiles $\left(17 \leq \mathrm{HepG}_{2} \mathrm{CC}_{50} \leq 53 \mu \mathrm{M}\right)$ and promising selectivity indexes $(11.3 \leq \mathrm{SI} \leq 27.5)$, in comparison with the antimalarial drug references chloroquine and doxycycline. Structure-activity relationships showed that the $\mathrm{CCl}_{3}$ group was mandatory for providing antiplasmodial activity to the pyrrolo[1,2-a]quinoxaline scaffold of the studied series. They also pointed out that the attachment of a pyrrole moiety to the quinoxaline ring improved the cytotoxicity profile without disturbing the anti-infectious activity. It finally showed that the substitution of the 4-trichloromethylpyrrolo[1,2a]quinoxaline scaffold at position 7 was neither necessary nor beneficial, as regards of both cytotoxicity or solubility in the biological media. Complementary pharmacomodulation works will be carried out to study the influence of the substitution of the same scaffold at other positions toward antiplasmodial activity.

\section{Experimental}

\subsection{Chemistry}


Commercial reagents were used as received without additional purification. Melting points were determined on a Kofler bench and are uncorrected. Elemental analysis and HRMS were carried out at the Spectropole, Faculté des Sciences et Techniques de Saint-Jérôme, Marseille, France. NMR spectra were recorded on a Bruker ARX 200 spectrometer at the Faculté de Pharmacie de Marseille $\left({ }^{1} \mathrm{H}-\mathrm{NMR}: 200 \mathrm{MHz},{ }^{13} \mathrm{C}-\mathrm{NMR}\right.$ : $\left.50 \mathrm{MHz}\right)$ or on aJEOL Lambda 400 Spectrometer at the Centre d'Etudes et de Recherche sur le Médicament de Normandie $\left({ }^{1} \mathrm{H}-\mathrm{NMR}: \quad 400 \mathrm{MHz},{ }^{13} \mathrm{C}-\mathrm{NMR}: 100 \mathrm{MHz}\right)$. NMR references were the following: ${ }^{1} \mathrm{H}: \mathrm{CHCl}_{3} \delta=7.26$, DMSO- $d_{6} \delta=2.50$ and ${ }^{13} \mathrm{C}: \mathrm{CHCl}_{3} \delta=76.9$, DMSO- $d_{6} \delta=$ 39.5. Solvents were dried by conventional methods. The following adsorbent was used for column chromatography: silica gel 60 (Merck, particle size 0.063-0.200 mm, 70-230 mesh ASTM). TLC was performed on $5 \mathrm{~cm} \times 10 \mathrm{~cm}$ aluminium plates coated with silica gel $60 \mathrm{~F}$ 254 (Merck) in an appropriate eluent. Visualization was made with ultraviolet light $(234 \mathrm{~nm})$. HRMS spectra were recorded on QStar Elite (Applied Biosystems SCIEX) spectrometer. PEG was the matrix for HRMS. The experimental exact mass was given for the ion which has the maximum isotopic abundance. Purity of synthetized compounds was checked with LC-MS analyses which were realized at the Faculté de Pharmacie de Marseille with a Thermo Scientific Accela High Speed LC System ${ }^{\circledR}$ coupled with a single quadrupole mass spectrometer Thermo MSQ Plus ${ }^{\circledR}$. The RP-HPLC column used is a Thermo Hypersil Gold ${ }^{\circledR}$ $50 \times 2.1 \mathrm{~mm}(\mathrm{C} 18$ bounded), with particles of $1.9 \mu \mathrm{m}$ diameter. The volume of sample injected on the column was $1 \mu \mathrm{L}$. The chromatographic analysis, total duration of $8 \mathrm{~min}$, is made with the gradient of following solvents: $\mathrm{t}=0 \mathrm{~min}$, water/methanol 50/50; $0<\mathrm{t}<4 \mathrm{~min}$, linear increase in the proportion of water to a ratio water/methanol 95/5; $4<\mathrm{t}<6 \mathrm{~min}$, water/methanol 95/5; $6<\mathrm{t}<7 \mathrm{~min}$, linear decrease in the proportion of water to return to a ratio 50/50 water/methanol; $6<\mathrm{t}<7 \mathrm{~min}$, water/methanol 50/50. The water used was buffered with $5 \mathrm{mM}$ ammonium acetate. The retention times $\left(t_{\mathrm{R}}\right)$ of the molecules analyzed are indicated in min. The preparation of compounds1a [21, 35], 3a [24, 26], 4b [22], 5a [21], 11a [37,38] and 12a [33] was achieved as described in the literature. Compounds $\mathbf{1 b}, \mathbf{2 b}, \mathbf{5 b}$, $\mathbf{5 d}$ and 5e were purchased from Sigma-Aldrich.

\subsubsection{1-(Trichloromethyl)isoquinoline (2a)}

A $100-\mathrm{mL}$ round-bottomed flask equipped with a drying tube-condenser and a magnetic stir bar was charged with 1-methylisoquinoline (500 mg, 3.49 mmol), $\mathrm{PCl}_{5}$ (4.40 g, $20.94 \mathrm{mmol}$ ) and a minimal amount of $\mathrm{POCl}_{3}$ to allow the stirring of the mixture. The reaction mixture was heated during $1 \mathrm{~h}$ under microwave heating $\left(800 \mathrm{~W}, 110^{\circ} \mathrm{C}\right)$. After the reaction mixture was 
cooled with an ice bath, it was carefully poured into $50 \mathrm{~mL}$ of ice and was made alkaline $(\mathrm{pH}$ 8) by addition of $\mathrm{NaHCO}_{3}$. Aqueous layer was extracted three times with $\mathrm{CH}_{2} \mathrm{Cl}_{2}$, and then dried with anhydrous sodium sulphate and finally concentrated under vacuum. Purification by chromatography on silica gel (Petroleum Ether/ $\mathrm{CH}_{2} \mathrm{Cl}_{2}$ ) gave the expected 1(trichloromethyl)isoquinoline.

Yield $24 \%$. Yellow powder. Mp $66^{\circ} \mathrm{C} .{ }^{1} \mathrm{H}$ NMR $\left(200 \mathrm{MHz}, \mathrm{CDCl}_{3}\right) \delta=8.88(\mathrm{dd}, J=1.8 ; 7.4$ Hz, 1H, H-3), 8.53 (d, J = 5.4 Hz, 1H, H-8), 7.94 (dd, $J=2.2 ; 7.2$ Hz, 1H, H-5), 7.67-7.80 (m, $3 \mathrm{H}, \mathrm{H}-4, \mathrm{H}-6$ and H-7). ${ }^{13} \mathrm{C}$ NMR (50 MHz, $\left.\mathrm{CDCl}_{3}\right) \delta=153.9,139.0,138.1,130.3,127.9$, 127.4, 127.1, 124.3, 123.3, 98.2. Anal. Calcd for $\mathrm{C}_{10} \mathrm{H}_{6} \mathrm{Cl}_{3} \mathrm{~N}$ : C, 48.72; H, 2.45; N, 5.68. Found: C, 48.32; H, 2.41; N, 5.64.

\subsubsection{2-(Trichloromethyl)quinazoline (4a)}

A 100-mL round-bottomed flask equipped with a drying tube-condenser and a magnetic stir bar was charged with 2-methylquinazoline [23] (200 mg, $1.4 \mathrm{mmol}), \mathrm{PCl}_{5}$ (870 mg, 4.2 mmol) and a minimal amount of $\mathrm{POCl}_{3}$ to allow the stirring of the mixture. The reaction mixture was heated during 15 min under microwave irradiation $\left(800 \mathrm{~W}, 150{ }^{\circ} \mathrm{C}\right)$. After the reaction mixture was cooled with an ice bath, it was carefully poured into $50 \mathrm{~mL}$ of ice and was made alkaline $(\mathrm{pH} 8)$ by addition of $\mathrm{NaOH}$. Aqueous layer was extracted twice with $\mathrm{CH}_{2} \mathrm{Cl}_{2}$, the organic layers were washed twice with brine and then dried with anhydrous sodium sulphate and finally concentrated under vacuum. Purification by chromatography on silica gel (Petroleum Ether/ $\mathrm{CH}_{2} \mathrm{Cl}_{2}$ ) gave the expected 2-(trichloromethyl)quinazoline.

Yield 30\%. Off-white powder. Mp $116^{\circ} \mathrm{C} .{ }^{1} \mathrm{H}$ NMR $\left(200 \mathrm{MHz}, \mathrm{CDCl}_{3}\right) \delta=9.57(\mathrm{~s}, 1 \mathrm{H}, \mathrm{H}-4)$, $8.22(\mathrm{~d}, J=9.1 \mathrm{~Hz}, 1 \mathrm{H}, \mathrm{H}-8), 8.01-8.08$ (m, 2H, H-5 and H-7), $7.81(\mathrm{dt}, J=1.2 ; 6.7 \mathrm{~Hz}, 1 \mathrm{H}$, H-6). ${ }^{13} \mathrm{C} \mathrm{NMR}\left(50 \mathrm{MHz} \mathrm{CDCl}_{3}\right) \delta=161.8,161.3,149.3,135.3,129.7,129.3,127.2,123.8$, 97.0. HRMS (ESI): $m / z[\mathrm{M}+\mathrm{H}]^{+}$calcd for $\left[\mathrm{C}_{9} \mathrm{H}_{6} \mathrm{Cl}_{3} \mathrm{~N}_{2}\right]^{+}$: 246.9596; found: 246.9599 .

\subsubsection{2-(Dichloromethyl)quinoxaline (5c)}

A 100-mL round-bottomed flask equipped with a drying tube-condenser and a magnetic stir bar was charged with 2-methylquinoxaline (2.0 g, $13.87 \mathrm{mmol}^{2}, \mathrm{POCl}_{3}(2.59 \mathrm{~mL}, 27.74$ $\mathrm{mmol})$ and $\mathrm{PCl}_{5}(5.78 \mathrm{~g}, 27.74 \mathrm{mmol})$. The mixture was refluxed for $12 \mathrm{~h}$. After the reaction mixture was cooled with an ice bath, the mixture was carefully poured into $200 \mathrm{~mL}$ of ice. The mixture was made alkaline by addition of $\mathrm{NaHCO}_{3}$. The resulting aqueous solution was extracted three times with $\mathrm{CHCl}_{3}$, and then the organic layer was dried with anhydrous 
sodium sulphate and was concentrated under vacuum. Purification by chromatography on silica gel $\left(\mathrm{CHCl}_{3}\right)$ gave the expected 2-(dichloromethyl)quinazoline.

Yield 13\%. Off-white powder. $\mathrm{Mp} 117^{\circ} \mathrm{C} .{ }^{1} \mathrm{H}$ NMR $\left(200 \mathrm{MHz}, \mathrm{CDCl}_{3}\right) \delta=9.33(\mathrm{~s}, 1 \mathrm{H}, \mathrm{H}-4)$, 8.18-8.05 (m, 2H, H-5 and H-8), 7.87-7.78 (m, 2H, H-6 and H-7), $6.88\left(\mathrm{~s}, 1 \mathrm{H}, \mathrm{CHCl}_{2}\right) .{ }^{13} \mathrm{C}$ NMR $\left(50 \mathrm{MHz}, \mathrm{CD}_{2} \mathrm{Cl}_{2}\right) \delta=151.9,143.3,142.5,140.1,131.2,131.0,129.5,129.3,70.2$. Anal. Calcd for $\mathrm{C}_{9} \mathrm{H}_{6} \mathrm{Cl}_{2} \mathrm{~N}_{2}:$ C, 50.73; H, 2.84; N, 13.15. Found: C, 50.66; H, 2.99; N, 12.92 .

\subsubsection{2-Phenyl-3-(trichloromethyl)quinoxaline $(5 f)$}

A 50-mL round-bottomed flask equipped with a drying tube-condenser and a magnetic stir bar was charged with 2-methyl-3-phenylquinoxaline [27] (441 mg, $2.0 \mathrm{mmol}), \mathrm{POCl}_{3}(1.86 \mathrm{~mL}$, $20.0 \mathrm{mmol})$ and $\mathrm{PCl}_{5}(2.08 \mathrm{~g}, 10.0 \mathrm{mmol})$. The mixture was refluxed for $1 \mathrm{~h} 30$. After the reaction mixture was cooled with an ice bath, the mixture was carefully poured into $200 \mathrm{~mL}$ of ice. The mixture was extracted three times with EtOAc. Then the organic layer was dried with anhydrous sodium sulphate and was concentrated under vacuum. Purification by chromatography on silica gel (Petroleum Ether/EtOAc) gave the expected 2-phenyl-3(trichloromethyl)quinoxaline.

Yield 26\%. White powder. Mp $125^{\circ} \mathrm{C} .{ }^{1} \mathrm{H}$ NMR $\left(200 \mathrm{MHz}, \mathrm{CDCl}_{3}\right) \delta=8.31-8.15(\mathrm{~m}, 2 \mathrm{H}, \mathrm{H}-$ 2'), 7.95-7.83 (m, 2H, H-5 and H-8), 7.64 (dd, $J=2.9$; 6.8 Hz, 2H, H-6 and H-7), 7.54- 7.44 (m, 3H, H-3' and H-4'). ${ }^{13} \mathrm{C}$ NMR (50 MHz, $\left.\mathrm{CDCl}_{3}\right) \delta=152.6,149.7$, 141.6, 139.6, 138.6, 132.3, 131.3, 129.8, 129.8, 129.0, 129.0, 127.9, 97.1. HRMS (ESI): $\mathrm{m} / z . \mathrm{M}+\mathrm{Na}]^{+}$calcd for $\left[\mathrm{C}_{15} \mathrm{H}_{9} \mathrm{Cl}_{3} \mathrm{~N}_{2} \mathrm{Na}\right]^{+}$: 344.9723; found: 344.9723 .

\subsubsection{Representative procedure for the preparation of 1-(4-substituted-2- nitrophenyl)-1H-pyrrole (7a-i)}

A $100-\mathrm{mL}$ round-bottomed single-neck flask equipped with a condenser and a magnetic stir bar was charged with appropriated 4-substituted-2-nitroaniline (25.0 mmol), 2,5dimethoxytetrahydofurane $(25.0 \mathrm{mmol})$ and $40 \mathrm{~mL}$ of glacial acetic acid. The flask was refluxed until disappearance of starting material (ca. $2 \mathrm{~h}$ ). After the reaction mixture was cooled to rt, removal of the volatiles under reduced pressure gave the crude product which was purified by column-chromatography (Petroleum Ether/EtOAc $=9: 1$ then 7:3) to give the desired 1-(4-substituted-2-nitrophenyl)-1H-pyrrole.

\subsubsection{1-(4-Iodo-2-nitrophenyl)- $1 H$-pyrrole (7e)}


Yield $82 \%$. Brown powder. Mp $70^{\circ} \mathrm{C} .{ }^{1} \mathrm{H}$ NMR $\left(400 \mathrm{MHz}, \mathrm{CDCl}_{3}\right) \delta=8.15(\mathrm{~d}, J=1.7 \mathrm{~Hz}$, 1H, H-3'), 7.96 (ddd, $J=0.7 ; 2.0 ; 8.6 \mathrm{~Hz}, 1 \mathrm{H}, \mathrm{H}-5$ '), 7.21 (d, $J=8.2 \mathrm{~Hz}, 1 \mathrm{H}, \mathrm{H}-6$ '), 6.76 (t, $J$ $=1.7 \mathrm{~Hz}, 2 \mathrm{H}, \mathrm{H}-2), 6.37(\mathrm{t}, J=2.0 \mathrm{~Hz}, 2 \mathrm{H}, \mathrm{H}-3) .{ }^{13} \mathrm{C} \mathrm{NMR}\left(100 \mathrm{MHz}, \mathrm{CDCl}_{3}\right) \delta=145.5$, 142.2, 134.0, 133.6, 129.3, 121.3, 111.7, 90.6. HRMS (ESI): $m / z[\mathrm{M}+\mathrm{H}]^{+}$calcd for $\left[\mathrm{C}_{10} \mathrm{H}_{8} \mathrm{~N}_{2} \mathrm{O}_{2} \mathrm{I}\right]^{+}:$: 314.9625; found: 314.9626 .

\subsubsection{Methyl 3-nitro-4-(1H-pyrrol-1-yl)benzoate (7i)}

Yield 79\%. Yellow powder. Mp $118^{\circ} \mathrm{C} .{ }^{1} \mathrm{H}$ NMR $\left(200 \mathrm{MHz}, \mathrm{CDCl}_{3}\right) \delta=8.47(\mathrm{~d}, J=1.5 \mathrm{~Hz}$, $1 \mathrm{H}, \mathrm{H}-2), 8.28$ (dd, $J=1.7 ; 8.4 \mathrm{~Hz}, 1 \mathrm{H}, \mathrm{H}-6), 7.54$ (d, $J=8.4 \mathrm{~Hz}, 1 \mathrm{H}, \mathrm{H}-5), 6.81$ (t, $J=2.1$ $\mathrm{Hz}, 2 \mathrm{H}, \mathrm{H}-2^{\prime}$ ), 6.39 (t, $J=2.1 \mathrm{~Hz}, 2 \mathrm{H}, \mathrm{H}-3$ ') 3.98 (s, 3H, $\left.\mathrm{CH}_{3}\right) .{ }^{13} \mathrm{C} \mathrm{NMR}\left(50 \mathrm{MHz}, \mathrm{CDCl}_{3}\right)$ $\delta=164.5,144.4,137.4,134.0,129.2,127.3,126.5,121.0,112.2,53.0$. HRMS (ESI): $m / z$ [M + $\mathrm{H}]^{+}$calcd for $\left[\mathrm{C}_{12} \mathrm{H}_{11} \mathrm{~N}_{2} \mathrm{O}_{4}\right]^{+}: 247.0713$; found: 247.0714 .

\subsubsection{Representative procedure for the preparation of 5-substituted-2-(1H- pyrrol-1-yl)aniline}

A 250-mL round-bottomed single-neck flask equipped with a condenser and a magnetic stir bar was charged with appropriated 1-(4-substituted-2-nitrophenyl)- $1 H$-pyrrole (15.0 mmol), tin (II) chloride dihydrate $(16.92 \mathrm{~g}, 75.0 \mathrm{mmol})$ and $60 \mathrm{~mL}$ of ethanol. The flask was refluxed until disappearance of starting material (ca. $3 \mathrm{~h}$ ). After the reaction mixture was cooled to rt, the mixture was quenched with a saturated solution of sodium carbonate. The white precipitate was filtered through a pad of Celite ${ }^{\circledR}$ and was thoroughly washed with ethanol. Then, removal of the volatiles under reduced pressure gave the crude 5-substituted-2-(1Hpyrrol-1-yl)aniline which was engaged in the next reaction step without any further purification

\subsubsection{5-Iodo-2-(1H-pyrrol-1-yl)aniline (8e)}

Yield 71\%. Orange powder. $\mathrm{Mp} 80^{\circ} \mathrm{C} .{ }^{1} \mathrm{H} \mathrm{NMR}\left(400 \mathrm{MHz}, \mathrm{CDCl}_{3}\right) \delta=7.15(\mathrm{~d}, J=1.7 \mathrm{~Hz}$, 1H, H-6), 7.09 (dd, $J=2.0 ; 8.1 \mathrm{~Hz}, 1 \mathrm{H}, \mathrm{H}-4), 6.85$ (d, $J=8.0 \mathrm{~Hz}, 1 \mathrm{H}, \mathrm{H}-3), 6.80$ (t, $J=1.7$ $\left.\mathrm{Hz}, 2 \mathrm{H}, \mathrm{H}-2^{\prime}\right), 6.34$ (t, $J=2.0 \mathrm{~Hz}, 2 \mathrm{H}, \mathrm{H}-3$ ') $\left.3.75\left(\mathrm{sl}, \mathrm{NH}_{2}\right) .{ }^{13} \mathrm{C} \mathrm{NMR} \mathrm{(100} \mathrm{MHz,} \mathrm{CDCl}_{3}\right) \delta$ $=143.5,128.7,127.4,127.2,124.7,121.6,109.9,93.4$. HRMS $(\mathrm{ESI}): m / z[\mathrm{M}+\mathrm{H}]^{+}$calcd for $\left[\mathrm{C}_{10} \mathrm{H}_{10} \mathrm{~N}_{2} \mathrm{I}\right]^{+}:$284.9883; found: 284.9884 .

\subsubsection{Methyl 3-amino-4-(1H-pyrrol-1-yl)benzoate (8i)}

Yield 52\%. Brown powder. Mp 80 ${ }^{\circ}$. ${ }^{1} \mathrm{H}$ NMR $\left(200 \mathrm{MHz}, \mathrm{CDCl}_{3}\right) \delta=7.52-7.39(\mathrm{~m}, 2 \mathrm{H}, \mathrm{H}-2$ and H-6), 7.18 (d, $J=8.0 \mathrm{~Hz}, 1 \mathrm{H}, \mathrm{H}-5), 6.87$ (t, $J=2.1 \mathrm{~Hz}, 2 \mathrm{H}, \mathrm{H}-2^{\prime}$ ) , 6.36 (t, $J=2.1 \mathrm{~Hz}, 2 \mathrm{H}$, 
$\left.\mathrm{H}-3{ }^{\prime}\right), 3.91\left(\mathrm{~s}, 3 \mathrm{H}, \mathrm{CH}_{3}\right) .{ }^{13} \mathrm{C}$ NMR $\left(50 \mathrm{MHz} \mathrm{CDCl}_{3}\right) \delta=166.8,141.7,131.1,130.0,126.8$, 121.4, 119.8, 117.4, 110.1, 52.3. HRMS (ESI): $m / z[\mathrm{M}+\mathrm{H}]^{+}$calcd for $\left[\mathrm{C}_{12} \mathrm{H}_{11} \mathrm{~N}_{2} \mathrm{O}_{2}\right]^{-}$: 215.0826; found: 215.0825 .

\subsubsection{Representative procedure for the preparation of 2,2,2-trichloro- $N$-[5- substituted-2-(1H-pyrrol-1-yl)phenyl]acetamide (9a-i)}

A 100-mL round-bottomed flask equipped with a condenser, an Ar inlet and a magnetic stir bar was charged with appropriated 5-substituted-2-(1H-pyrrol-1-yl)aniline (15.0 mmol), 25 $\mathrm{mL}$ of anhydrous dioxane, triethylamine $(3.14 \mathrm{~mL}, 22.5 \mathrm{mmol})$. The mixture was cooled with a ice bath and trichloroacetyl chloride was added dropwise $(1.67 \mathrm{~mL}, 15.0 \mathrm{mmol})$. The flask was refluxed until disappearance of starting material (ca. $4 \mathrm{~h}$ ). After the reaction mixture was cooled to rt, removal of the volatiles under reduced pressure gave the crude product which was dissolved in EtOAc. The organic layer was washed with a aqueous solution of sodium bicarbonate and brine, then the organic layer was dried with anhydrous sodium sulphate and was concentrated under vacuum. Purification by chromatography on silica gel (Petroleum Ether/EtOAc) gave the expected 2,2,2-trichloro- $N$-[5-substituted-2-(1H-pyrrol-1yl)phenyl]acetamide.

\subsubsection{1. $\quad N$-[2-(1H-Pyrrol-1-yl)phenyl]-2,2,2-trichloroacetamide (9a)}

Yield 65\%. Off-white powder. $\mathrm{Mp} 79^{\circ} \mathrm{C} .{ }^{1} \mathrm{H}$ NMR $\left(200 \mathrm{MHz}, \mathrm{CDCl}_{3}\right) \delta=8.41(\mathrm{~m}, 1 \mathrm{H}, \mathrm{H}-6)$, 8.37 (bs, NH), 7.48 (dt, $J=1.8 ; 8.4 \mathrm{~Hz}, 1 \mathrm{H}, \mathrm{H}-5), 7.37$ (dt, $J=1.6 ; 7.8 \mathrm{~Hz}, 1 \mathrm{H}, \mathrm{H}-3$ ), 7.287.24 (m, 1H, H-4), 6.82 (t, $J=2.2 \mathrm{~Hz}, 2 \mathrm{H}, \mathrm{H}-2$ pyrrole), 6.43 (t, $J=2.2 \mathrm{~Hz}, 2 \mathrm{H}, \mathrm{H}-3$ pyrrole).

${ }^{13} \mathrm{C} \mathrm{NMR}\left(50 \mathrm{MHz}, \mathrm{CDCl}_{3}\right) \delta=159.3,132.4,131.8,129.2,127.3,125.9,122.1,120.8,111.2$, 92.6. HRMS (ESI): $\mathrm{m} / z[\mathrm{M}+\mathrm{H}]^{+}$calcd for $\left[\mathrm{C}_{12} \mathrm{H}_{10} \mathrm{~N}_{2} \mathrm{OCl}_{3}\right]^{+}: 302.9853$; found: 302.9852 .

\subsubsection{2,2,2-Trichloro- $N$-[5-fluoro-2-(1H-pyrrol-1-yl)phenyl]acetamide} (9b)

Yield 65\%. Brown powder. Mp 64 ${ }^{\circ} \mathrm{C} .{ }^{1} \mathrm{H}$ NMR (400 MHz, $\left.\mathrm{CDCl}_{3}\right) \delta=8.34$ (bs, NH), 6.85 (dd, $J=2.7 ; 10.2 \mathrm{~Hz}, 1 \mathrm{H}, \mathrm{H}-6), 7.36$ (dd, $J=5.6 ; 8.8 \mathrm{~Hz}, 1 \mathrm{H}, \mathrm{H}-3), 6.98$ (td, $J=2.9 ; 7.8 \mathrm{~Hz}$, $1 \mathrm{H}, \mathrm{H}-4), 6.78$ (t, $J=2.0 \mathrm{~Hz}, 2 \mathrm{H}, \mathrm{H}-2$ pyrrole), $6.43\left(\mathrm{t}, J=2.0 \mathrm{~Hz}, 2 \mathrm{H}, \mathrm{H}-3\right.$ pyrrole) ${ }^{13} \mathrm{C}$ NMR $\left(100 \mathrm{MHz}, \mathrm{CDCl}_{3}\right) \delta=162.4(\mathrm{~d}, J=266.8 \mathrm{~Hz}), 159.3,134.1(\mathrm{~d}, J=12.3 \mathrm{~Hz}), 128.6(\mathrm{~d}$, $J=9.9 \mathrm{~Hz}), 127.5,122.3,112.5(\mathrm{~d}, J=23.0 \mathrm{~Hz}), 111.5,108.1(\mathrm{~d}, J=29.6 \mathrm{~Hz}), 92.3$. HRMS (ESI): $m / z[\mathrm{M}+\mathrm{H}]^{+}$calcd for $\left[\mathrm{C}_{12} \mathrm{H}_{9} \mathrm{~N}_{2} \mathrm{OFCl}_{3}\right]^{+}: 320.9759$; found: 320.9762 .

\subsubsection{2,2,2-Trichloro- $N$-[5-chloro-2-(1H-pyrrol-1-yl)phenyl]acetamide} (9c) 
Yield 63\%. Yellow powder. Mp $98^{\circ} \mathrm{C}$. ${ }^{1} \mathrm{H}$ NMR $\left(400 \mathrm{MHz}, \mathrm{CDCl}_{3}\right) \delta=8.43(\mathrm{~d}, J=2.2 \mathrm{~Hz}$, 1H, H-6), 8.29 (bs, NH), 7.26 (d, $J=8.6 \mathrm{~Hz}, 1 \mathrm{H}, \mathrm{H}-3), 7.20$ (dd, $J=2.2 ; 8.6 \mathrm{~Hz}, 1 \mathrm{H}, \mathrm{H}-4$ ), $6.73\left(\mathrm{t}, J=1.7 \mathrm{~Hz}, 2 \mathrm{H}, \mathrm{H}-2\right.$ pyrrole), $6.38\left(\mathrm{t}, J=1.7 \mathrm{~Hz}, 2 \mathrm{H}, \mathrm{H}-3\right.$ pyrrole) ${ }^{13} \mathrm{C}$ NMR (100 $\left.\mathrm{MHz}, \mathrm{CDCl}_{3}\right) \delta=159.3,135.1,133.4,130.0,128.2,125.9,122.1,120.8,111.6,92.3$. HRMS (ESI): $m / z$ [M - H] $]^{-}$calcd for $\left[\mathrm{C}_{12} \mathrm{H}_{7} \mathrm{~N}_{2} \mathrm{OCl}_{4}\right]^{-}: 336.9289$; found: 336.9288 .

\subsubsection{4. $N$-[5-Bromo-2-(1H-pyrrol-1-yl)phenyl]-2,2,2-trichloroacetamide} (9d)

Yield 63\%. Beige powder. Mp 90 ${ }^{\circ} \mathrm{C} .{ }^{1} \mathrm{H}$ NMR $\left(400 \mathrm{MHz}, \mathrm{CDCl}_{3}\right) \delta=8.57(\mathrm{~d}, J=2.0 \mathrm{~Hz}$, 1H, H-6), 8.29 (bs, NH), 7.37 (dd, $J=2.2 ; 8.6 \mathrm{~Hz}, 1 \mathrm{H}, \mathrm{H}-3), 7.20$ (d, $J=8.6 \mathrm{~Hz}, 1 \mathrm{H}, \mathrm{H}-4$ ), $6.73\left(\mathrm{t}, J=1.7 \mathrm{~Hz}, 2 \mathrm{H}, \mathrm{H}-2\right.$ pyrrole), 6.38 (t, $J=1.7 \mathrm{~Hz}, 2 \mathrm{H}, \mathrm{H}-3$ pyrrole). ${ }^{13} \mathrm{C}$ NMR (100 $\left.\mathrm{MHz}, \mathrm{CDCl}_{3}\right) \delta=159.3,133.5,130.6,128.9,128.5,123.7,122.8,122.0,111.7,92.3$. HRMS (ESI): $m / z[\mathrm{M}+\mathrm{H}]^{+}$calcd for $\left[\mathrm{C}_{12} \mathrm{H}_{9} \mathrm{~N}_{2} \mathrm{OBrCl}_{3}\right]^{+}: 382.8934$; found: 382.8933 .

\subsubsection{2,2,2-Trichloro- $N$-[5-iodo-2-(1H-pyrrol-1-yl)phenyl]acetamide (9e)}

Yield 69\%. Brown powder. Mp 90 ${ }^{\circ} \mathrm{C} .{ }^{1} \mathrm{H}$ NMR $\left(400 \mathrm{MHz}, \mathrm{CDCl}_{3}\right) \delta=8.79(\mathrm{~d}, J=1.7 \mathrm{~Hz}$, 1H, H-6), 8.32 (bs, NH), 7.63 (dd, $J=1.9 ; 8.3 \mathrm{~Hz}, 1 \mathrm{H}, \mathrm{H}-4), 7.10$ (d, $J=8.3 \mathrm{~Hz}, 1 \mathrm{H}, \mathrm{H}-3$ ), $6.79\left(\mathrm{t}, J=1.9 \mathrm{~Hz}, 2 \mathrm{H}, \mathrm{H}-2\right.$ pyrrole), $6.43\left(\mathrm{t}, J=1.9 \mathrm{~Hz}, 2 \mathrm{H}, \mathrm{H}-3\right.$ pyrrole). ${ }^{13} \mathrm{C}$ NMR (100 $\left.\mathrm{MHz}, \mathrm{CDCl}_{3}\right) \delta=159.3,135.1,133.3,131.3,129.5,128.6,122.0,111.6,93.8,82.3 . \mathrm{HRMS}$ (ESI): $m / z[\mathrm{M}+\mathrm{H}]^{+}$calcd for $\left[\mathrm{C}_{12} \mathrm{H}_{9} \mathrm{~N}_{2} \mathrm{OICl}_{3}\right]^{+}: 428.8820$; found: 428.8818 .

\subsubsection{2,2,2-Trichloro- $N$-(5-methyl-2-(1H-pyrrol-1-yl)phenyl)acetamide} (9f)

Yield 46\%. Amber oil. ${ }^{1} \mathrm{H}$ NMR $\left(200 \mathrm{MHz}, \mathrm{CDCl}_{3}\right) \delta=8.31$ (bs, NH), 8.23 (s, 1H, H-6), 7.26 (d, $J=8.0 \mathrm{~Hz}, 1 \mathrm{H}, \mathrm{H}-3), 7.09$ (d, $J=8.0 \mathrm{~Hz}, 1 \mathrm{H}, \mathrm{H}-4), 6.79$ (t, $J=2.1 \mathrm{~Hz}, 2 \mathrm{H}, \mathrm{H}-2$ pyrrole), $6.41\left(\mathrm{t}, J=2.1 \mathrm{~Hz}, 2 \mathrm{H}, \mathrm{H}-3\right.$ pyrrole), $2.46\left(\mathrm{~s}, 3 \mathrm{H}, \mathrm{CH}_{3}\right) .{ }^{13} \mathrm{C} \mathrm{NMR}\left(50 \mathrm{MHz}, \mathrm{CDCl}_{3}\right) \delta$ $=159.2,139.5,132.0,129.3,126.9,126.5,122.2,121.2,110.9,92.6,21.6 . \mathrm{HRMS}(\mathrm{ESI}): \mathrm{m} / \mathrm{z}$ $[\mathrm{M}+\mathrm{H}]^{+}$calcd for $\left[\mathrm{C}_{13} \mathrm{H}_{11} \mathrm{~N}_{2} \mathrm{OCl}_{3}\right]^{+}:$317.0010; found: 317.0013 .

\subsubsection{2,2,2-Trichloro- $N$-(5-methoxy-2-(1H-pyrrol-1-yl)phenyl)acetamide $(9 \mathrm{~g})$}

Yield $88 \%$. Yellow powder. Mp $67^{\circ} \mathrm{C} .{ }^{1} \mathrm{H}$ NMR $\left(200 \mathrm{MHz}, \mathrm{CDCl}_{3}\right) \delta=8.29$ (bs, NH), 8.04 (d, $J=2.8 \mathrm{~Hz}, 1 \mathrm{H}, \mathrm{H}-6), 7.28$ (d, $J=9.3 \mathrm{~Hz}, 1 \mathrm{H}, \mathrm{H}-3), 6.79$ (dd, $J=2.2 ; 8.2 \mathrm{~Hz}, 1 \mathrm{H}, \mathrm{H}-4)$, $6.76(\mathrm{t}, J=2.1 \mathrm{~Hz}, 2 \mathrm{H}, \mathrm{H}-2$ pyrrole), $6.40(\mathrm{t}, J=2.1 \mathrm{~Hz}, 2 \mathrm{H}, \mathrm{H}-3$ pyrrole), 3.88 (s, 3H, $\left.\mathrm{OCH}_{3}\right) .{ }^{13} \mathrm{C} \mathrm{NMR}\left(50 \mathrm{MHz}, \mathrm{CDCl}_{3}\right) \delta=159.9,159.2,133.5,128.0,124.3,122.4,111.6$, 
110.8, 105.1, 92.4, 55.8. HRMS (ESI): $m / z,[\mathrm{M}+\mathrm{H}]^{+}$calcd for $\left[\mathrm{C}_{13} \mathrm{H}_{12} \mathrm{~N}_{2} \mathrm{O}_{2} \mathrm{Cl}_{3}\right]^{+}: 332.9959$; found: 332.9958 .

\subsubsection{2,2,2-Trichloro- $N$-(5-cyano-2-(1H-pyrrol-1-yl)phenyl)acetamide} (9h)

Yield 77\%. Yellow powder. Mp $129^{\circ} \mathrm{C} .{ }^{1} \mathrm{H}$ NMR $\left(200 \mathrm{MHz}, \mathrm{CDCl}_{3}\right) \delta=8.75(\mathrm{~d}, J=1.6 \mathrm{~Hz}$, 1H, H-6), 8.54 (bs, NH), 7.59 (dd, $J=1.7 ; 8.2 \mathrm{~Hz}, 1 \mathrm{H}, \mathrm{H}-4), 7.48$ (d, $J=8.1 \mathrm{~Hz}, 1 \mathrm{H}, \mathrm{H}-3$ ), $6.84\left(\mathrm{t}, J=2.1 \mathrm{~Hz}, 2 \mathrm{H}, \mathrm{H}-2\right.$ pyrrole), 6.48 (t, $J=2.1 \mathrm{~Hz}, 2 \mathrm{H}, \mathrm{H}-3$ pyrrole). ${ }^{13} \mathrm{C}$ NMR (50 $\left.\mathrm{MHz}, \mathrm{CDCl}_{3}\right) \delta=159.5,135.1,132.8,129.6,127.9,124.5,121.7,117.6,112.8,112.4,92.1$. HRMS (ESI): $m / z,[\mathrm{M}+\mathrm{H}]^{+}$calcd for $\left[\mathrm{C}_{13} \mathrm{H}_{9} \mathrm{~N}_{3} \mathrm{OCl}_{3}\right]^{+}: 327.9806$; found: 327.9802 .

\subsubsection{Methyl 4-(1H-pyrrol-1-yl)-3-(2,2,2-trichloroacetamido)benzoate (9i)}

Yield 77\%. Amber oil. ${ }^{1} \mathrm{H}$ NMR $\left(200 \mathrm{MHz}, \mathrm{CDCl}_{3}\right) \delta=9.00(\mathrm{~d}, J=1.8 \mathrm{~Hz}, 1 \mathrm{H}, \mathrm{H}-6), 8.46$ (bs, NH), 7.98 (dd, $J=1.8 ; 8.2 \mathrm{~Hz}, 1 \mathrm{H}, \mathrm{H}-4), 7.44$ (d, $J=8.2 \mathrm{~Hz}, 1 \mathrm{H}, \mathrm{H}-3), 6.85$ (t, $J=2.1$ $\mathrm{Hz}, 2 \mathrm{H}, \mathrm{H}-2$ pyrrole), 6.45 (t, $J=2.1 \mathrm{~Hz}, 2 \mathrm{H}, \mathrm{H}-3$ pyrrole), $3.96\left(\mathrm{~s}, 3 \mathrm{H}, \mathrm{CH}_{3}\right) .{ }^{13} \mathrm{C}$ NMR (50 $\left.\mathrm{MHz}, \mathrm{CDCl}_{3}\right) \delta=165.9,159.4,135.5,131.8,130.7,127.5,127.0,112.5,121.8,111.8,92.4$, 52.7. HRMS (ESI): $m / z[\mathrm{M}+\mathrm{H}]^{+}$calcd for $\left[\mathrm{C}_{14} \mathrm{H}_{12} \mathrm{~N}_{2} \mathrm{O}_{3} \mathrm{Cl}_{3}\right]^{+}: 360.9908$; found: 360.9904 .

\subsubsection{Representative procedure for the preparation of 7-substituted-4- (trichloromethyl)pyrrolo[1,2-a]quinoxaline (10a-i)}

A 100-mL round-bottomed flask equipped with a condenser, an Ar inlet and a magnetic stir bar was charged with appropriated 2,2,2-trichloro- $N$-[5-substituted-2-(1H-pyrrol-1yl)phenyl]acetamide (5.0 mmol), $15 \mathrm{~mL}$ of freshly distilled $\mathrm{POCl}_{3}$ and anhydrous pyridine (1 $\mathrm{mL}$ ). The mixture was refluxed until disappearance of starting material (ca. $4 \mathrm{~h}$ ). After the reaction mixture was cooled with an ice bath, it was carefully poured over $200 \mathrm{~mL}$ of ice. The mixture was made alkaline with concentrated ammonia to $\mathrm{pH} 10$. The alkaline aqueous phase was extracted three times with $\mathrm{CH}_{2} \mathrm{Cl}_{2}$. The combined organic layers were washed with $10 \%$ hydrochloric acid and then by water. Then the organic layer was dried with anhydrous sodium sulphate and was concentrated under vacuum. Purification by chromatography on silica gel (Petroleum Ether/EtOAc) gave the expected 7-substituted-4-(trichloromethyl)pyrrolo[1,2a]quinoxaline.

\subsubsection{4-(Trichloromethyl)pyrrolo[1,2-a]quinoxaline (10a)}

Yield 57\%. Beige powder. Mp 202 ${ }^{\circ} \mathrm{C} .{ }^{1} \mathrm{H}$ NMR $\left(400 \mathrm{MHz}, \mathrm{CDCl}_{3}\right) \delta=8.10(\mathrm{dd}, J=1.4 ; 8.3$ Hz, 1H, H-6), 8.02 (dd, $J=1.0 ; 2.7 \mathrm{~Hz}, 1 \mathrm{H}, \mathrm{H}-1$ ), 7.90 (dd, $J=1.2 ; 8.3 \mathrm{~Hz}, 1 \mathrm{H}, \mathrm{H}-9), 7.63$ 
(dt, $J=1.2 ; 8.3 \mathrm{~Hz}, 1 \mathrm{H}, \mathrm{H}-8), 7.50$ (dt, $J=0.7 ; 7.3 \mathrm{~Hz}, 1 \mathrm{H}, \mathrm{H}-7), 7.40$ (dd, $J=0.7 ; 4.2 \mathrm{~Hz}$, $1 \mathrm{H}, \mathrm{H}-3), 6.98(\mathrm{dd}, J=2.7 ; 4.2 \mathrm{~Hz}, 1 \mathrm{H}, \mathrm{H}-2) .{ }^{13} \mathrm{C} \mathrm{NMR}\left(100 \mathrm{MHz}, \mathrm{CDCl}_{3}\right) \delta=150.1,133.5$, $131.5,130.0,127.8,125.8,120.6,115.2,114.2,113.8,110.4,96.3$. Anal. Calcd for $\mathrm{C}_{12} \mathrm{H}_{7} \mathrm{Cl}_{3} \mathrm{~N}_{2}$ : C, 50.47; H, 2.47; N, 9.81. Found: C, 50.35; H, 2.37; N, 9.67.

\subsubsection{7-Fluoro-4-(trichloromethyl)pyrrolo[1,2-a]quinoxaline (10b)}

Yield 56\%. Yellow powder. Mp $192^{\circ} \mathrm{C} .{ }^{1} \mathrm{H}$ NMR $\left(400 \mathrm{MHz}, \mathrm{CDCl}_{3}\right) \delta=7.97(\mathrm{dd}, J=1.2$; $2.7 \mathrm{~Hz}, 1 \mathrm{H}, \mathrm{H}-1), 7.85$ (dd, $J=4.9 ; 9.0 \mathrm{~Hz}, 1 \mathrm{H}, \mathrm{H}-6$ ), 7.77 (dd, $J=2.9 ; 9.0 \mathrm{~Hz}, 1 \mathrm{H}, \mathrm{H}-9$ ), 7.41-7.34 (m, 2H, H-3 and H-8), 6.97 (dd, $J=2.7$; 4.1 Hz, 1H, H-2). ${ }^{13} \mathrm{C}$ NMR (100 MHz, $\left.\mathrm{CDCl}_{3}\right) \delta=160.0(\mathrm{~d}, J=244.3 \mathrm{~Hz}), 151.1,134.6(\mathrm{~d}, J=11.5 \mathrm{~Hz}), 124.4,120.4,117.9(\mathrm{~d}, J=$ $23.9 \mathrm{~Hz}), 116.6(\mathrm{~d}, J=23.0 \mathrm{~Hz}), 115.4,115.0(\mathrm{~d}, J=9.1 \mathrm{~Hz}), 114.4,110.7$, 96.2. Anal. Calcd for $\mathrm{C}_{12} \mathrm{H}_{6} \mathrm{Cl}_{3} \mathrm{FN}_{2}$ : C, 47.48; H, 1.99; N, 9.23. Found: C, 47.81; H, 1.76; N, 9.10.

\subsubsection{7-Chloro-4-(trichloromethyl)pyrrolo[1,2-a]quinoxaline (10c)}

Yield 52\%. Yellow-green powder. Mp $132{ }^{\circ} \mathrm{C} .{ }^{1} \mathrm{H}$ NMR $\left(400 \mathrm{MHz}, \mathrm{CDCl}_{3}\right) \delta=8.09(\mathrm{~d}, J=$ $2.2 \mathrm{~Hz}, 1 \mathrm{H}, \mathrm{H}-6), 7.97$ (dd, $J=1.2 ; 2.9 \mathrm{~Hz}, 1 \mathrm{H}, \mathrm{H}-1), 7.81$ (d, $J=8.8 \mathrm{~Hz}, 1 \mathrm{H}, \mathrm{H}-9), 7.57$ (dd, $J=2.4 ; 8.8 \mathrm{~Hz}, 1 \mathrm{H}, \mathrm{H}-8), 7.41$ (dd, $J=1.0 ; 4.2 \mathrm{~Hz}, 1 \mathrm{H}, \mathrm{H}-3), 6.98$ (dd, $J=2.7 ; 4.2 \mathrm{~Hz}, 1 \mathrm{H}$, $\mathrm{H}-2) .{ }^{13} \mathrm{C} \mathrm{NMR}\left(100 \mathrm{MHz}, \mathrm{CDCl}_{3}\right) \delta=151.1,134.4,131.0,130.8,130.0,126.4,120.5,115.5$, 115.0, 114.6, 110.9, 95.9. Anal. Calcd for $\mathrm{C}_{12} \mathrm{H}_{6} \mathrm{Cl}_{4} \mathrm{~N}_{2}$ : C, 45.04; H, 1.89; N, 8.75. Found: C, 45.44; H, 1.89; N, 8.75.

\subsubsection{7-Bromo-4-(trichloromethyl)pyrrolo[1,2-a]quinoxaline (10d)}

Yield 59\%. Yellow-green powder. $\mathrm{Mp} 156^{\circ} \mathrm{C} .{ }^{1} \mathrm{H}$ NMR $\left(400 \mathrm{MHz}, \mathrm{CDCl}_{3}\right) \delta=8.27(\mathrm{~d}, J=$ $2.2 \mathrm{~Hz}, 1 \mathrm{H}, \mathrm{H}-6), 7.99$ (dd, $J=1.0 ; 2.7 \mathrm{~Hz}, 1 \mathrm{H}, \mathrm{H}-1$ ), 7.77 (d, $J=8.8 \mathrm{~Hz}, 1 \mathrm{H}, \mathrm{H}-9), 7.72$ (dd, $J=2.2 ; 8.8 \mathrm{~Hz}, 1 \mathrm{H}, \mathrm{H}-8), 7.41(\mathrm{dd}, J=1.0 ; 4.2 \mathrm{~Hz}, 1 \mathrm{H}, \mathrm{H}-3), 6.99$ (dd, $J=2.7 ; 4.2 \mathrm{~Hz}, 1 \mathrm{H}$, $\mathrm{H}-2) .{ }^{13} \mathrm{C} \mathrm{NMR}\left(100 \mathrm{MHz}, \mathrm{CDCl}_{3}\right) \delta=151.0,134.7,133.9,132.9,126.8,120.6,118.3,115.5$, 115.2, 114.7, 111.0, 95.9. Anal. Calcd for $\mathrm{C}_{12} \mathrm{H}_{6} \mathrm{BrCl}_{3} \mathrm{~N}_{2}$ : C, 39.55; H, 1.66; N, 7.69. Found: C, 39.39; H, 1.74; N, 7.61.

\subsubsection{7-Iodo-4-(trichloromethyl)pyrrolo[1,2-a]quinoxaline (10e)}

Yield 56\%. Brown powder. Mp $170^{\circ} \mathrm{C} .{ }^{1} \mathrm{H}$ NMR $\left(400 \mathrm{MHz}, \mathrm{CDCl}_{3}\right) \delta=8.45(\mathrm{~d}, J=2.0 \mathrm{~Hz}$, 1H, H-6), 7.97-7.95 (m, 1H, H-1), 7.87 (dd, $J=1.7 ; 8.6 \mathrm{~Hz}, 1 \mathrm{H}, \mathrm{H}-8), 7.62$ (d, $J=8.8 \mathrm{~Hz}, 1 \mathrm{H}$, $\mathrm{H}-9), 7.40(\mathrm{~d}, J=4.2 \mathrm{~Hz}, 1 \mathrm{H}, \mathrm{H}-3), 6.98$ (dd, $J=2.7 ; 4.0 \mathrm{~Hz}, 1 \mathrm{H}, \mathrm{H}-2) .{ }^{13} \mathrm{C} \mathrm{NMR}(100 \mathrm{MHz}$, $\left.\mathrm{CDCl}_{3}\right) \delta=150.8,139.9,138.3,134.6,127.4,120.5,115.4,115.3,114.5,110.9,95.9,88.6$. Anal. Calcd for $\mathrm{C}_{12} \mathrm{H}_{6} \mathrm{ICl}_{3} \mathrm{~N}_{2}$ : C, 35.03; H, 1.47; N, 6.81. Found: C, 34.69; H, 1.49; N, 6.69. 


\subsubsection{7-Methyl-4-(trichloromethyl)pyrrolo[1,2-a]quinoxaline (10f)}

Yield $20 \%$. Yellow powder. Mp $136^{\circ} \mathrm{C} .{ }^{1} \mathrm{H}$ NMR $\left(200 \mathrm{MHz}, \mathrm{CDCl}_{3}\right) \delta=7.97(\mathrm{dd}, J=1.2$; $2.7 \mathrm{~Hz}, 1 \mathrm{H}, \mathrm{H}-1$ ), 7.90 (s, 1H, H-6), 7.78 (d, $J=8.4 \mathrm{~Hz}, 1 \mathrm{H}, \mathrm{H}-9), 7.44$ (dd, $J=1.6$; $8.4 \mathrm{~Hz}$, 1H, H-8), 7.37 (dd, $J=1.2 ; 4.2 \mathrm{~Hz}, 1 \mathrm{H}, \mathrm{H}-3$ ), 6.94 (dd, $J=2.8 ; 4.2 \mathrm{~Hz}, 1 \mathrm{H}, \mathrm{H}-2), 2.51$ (s, 3H, $\left.\mathrm{CH}_{3}\right) .{ }^{13} \mathrm{C} \mathrm{NMR}\left(50 \mathrm{MHz}, \mathrm{CDCl}_{3}\right) \delta=150.0,135.7,133.5,131.2,131.1,125.6,120.5,114.9$, 113.9, 113.5, 110.0, 96.6, 21.1. Anal. Calcd for $\mathrm{C}_{13} \mathrm{H}_{9} \mathrm{Cl}_{3} \mathrm{~N}_{2}$ : C, 52.12; H, 3.03; N, 9.35. Found: C, 52.18; H, 2.76; N, 9.60.

\subsubsection{7-Methoxy-4-(trichloromethyl)pyrrolo[1,2-a]quinoxaline (10g)}

Yield 60\%. Brown powder. $\mathrm{Mp} 108^{\circ} \mathrm{C} .{ }^{1} \mathrm{H}$ NMR $\left(200 \mathrm{MHz}, \mathrm{CDCl}_{3}\right) \delta=7.93(\mathrm{dd}, J=1.1 ; 2.6$ Hz, 1H, H-1), 7.79 (d, $J=9.1 \mathrm{~Hz}, 1 \mathrm{H}, \mathrm{H}-9), 7.52$ (d, $J=2.8 \mathrm{~Hz}, 1 \mathrm{H}, \mathrm{H}-6), 7.36$ (dd, $J=1.1$; $4.2 \mathrm{~Hz}, 1 \mathrm{H}, \mathrm{H}-3), 7.23$ (dd, $J=2.5 ; 8.8 \mathrm{~Hz}, 1 \mathrm{H}, \mathrm{H}-8), 6.93$ (dd, $J=2.7 ; 4.2 \mathrm{~Hz}, 1 \mathrm{H}, \mathrm{H}-2), 3.94$ $\left(\mathrm{s}, 3 \mathrm{H}, \mathrm{CH}_{3}\right) .{ }^{13} \mathrm{C} \mathrm{NMR}\left(50 \mathrm{MHz}, \mathrm{CDCl}_{3}\right) \delta=157.5,150.4,134.6,122.0,120.4,119.6,114.8$, 114.7, 113.9, 112.1, 109.9, 96.5, 56.0. Anal. Calcd for $\mathrm{C}_{13} \mathrm{H}_{9} \mathrm{Cl}_{3} \mathrm{~N}_{2} \mathrm{O}: \mathrm{C}, 49.48 ; \mathrm{H}, 2.87 ; \mathrm{N}$, 8.88. Found: C, 49.72; H, 3.22; N, 8.98.

\subsubsection{4-(Trichloromethyl)pyrrolo[1,2-a]quinoxaline-7-carbonitrile (10h)}

Yield $60 \%$. Beige powder. Mp $177^{\circ} \mathrm{C} .{ }^{1} \mathrm{H} \mathrm{NMR}\left(200 \mathrm{MHz}, \mathrm{CDCl}_{3}\right) \delta=8.40(\mathrm{~d}, J=1.6 \mathrm{~Hz}$, 1H, H-1), 8.04 (d, $J=2.6 \mathrm{~Hz}, 1 \mathrm{H}, \mathrm{H}-6), 7.97$ (d, $J=8.6 \mathrm{~Hz}, 1 \mathrm{H}, \mathrm{H}-9), 7.84$ (dd, $J=1.6$; 8.6 $\mathrm{Hz}, 1 \mathrm{H}, \mathrm{H}-8), 7.47$ (d, $J=4.2 \mathrm{~Hz}, 1 \mathrm{H}, \mathrm{H}-3), 7.08-7.05$ (m, 1H, H-2). ${ }^{13} \mathrm{C}$ NMR $(50 \mathrm{MHz}$, $\left.\mathrm{CDCl}_{3}\right) \delta=151.8,135.9,133.5,132.3,130.5,120.7,117.9,116.2,115.7,115.1,112.0,109.3$, 95.8. Anal. Calcd for $\mathrm{C}_{13} \mathrm{H}_{6} \mathrm{Cl}_{3} \mathrm{~N}_{3}$ : C, 50.28; H, 1.95; N, 13.53. Found: $\mathrm{C}, 50.63 ; \mathrm{H}, 2.02 ; \mathrm{N}$, 13.66 .

\subsubsection{Methyl 4-(trichloromethyl)pyrrolo[1,2-a]quinoxaline-7-carboxylate} (10i)

Yield $69 \%$. Beige powder. Mp $224{ }^{\circ} \mathrm{C} .{ }^{1} \mathrm{H}$ NMR $\left(200 \mathrm{MHz}, \mathrm{CDCl}_{3}\right) \delta=8.79(\mathrm{~d}, J=1.6 \mathrm{~Hz}$, 1H, H-6), 8.39-8.21 (m, 1H, H-8), 8.05 (d, $J=1.1 \mathrm{~Hz}, 1 \mathrm{H}, \mathrm{H}-1), 7.94$ (dd, $J=1.5 ; 8.7 \mathrm{~Hz}, 1 \mathrm{H}$, $\mathrm{H}-9), 7.44$ (d, $J=1.5 \mathrm{~Hz}, 1 \mathrm{H}, \mathrm{H}-3), 7.02$ (d, $J=4.2 \mathrm{~Hz}, 1 \mathrm{H}, \mathrm{H}-2), 3.99$ (s, 3H, $\left.\mathrm{CH}_{3}\right) .{ }^{13} \mathrm{C} \mathrm{NMR}$ $\left(50 \mathrm{MHz}, \mathrm{CDCl}_{3}\right) \delta=166.1,150.9,133.5,133.2,130.7,127.6,127.5,120.8,115.8,115.1$, 114.0, 11.3, 96.1, 52.6. Anal. Calcd for $\mathrm{C}_{14} \mathrm{H}_{9} \mathrm{Cl}_{3} \mathrm{~N}_{2} \mathrm{O}_{2}$ : C, 48.94; H, 2.64; N, 8.15. Found: C, $49.15 ; \mathrm{H}, 2.63 ; \mathrm{N}, 8.09$.

3.1.9. 4-(Trichloromethyl)pyrrolo[1,2-a]quinoxaline-7-carboxylic acid (10j) 
A 100-mL round-bottomed flask equipped with a condenser and a magnetic stir bar was charged with methyl 4-(trichloromethyl)pyrrolo[1,2-a]quinoxaline-7-carboxylate $(500 \mathrm{mg}$, $1.45 \mathrm{mmol}$ ) and $15 \mathrm{~mL}$ of conc. $\mathrm{HCl}$. The mixture was refluxed until disappearance of starting material (ca. $5 \mathrm{~h}$ ). After the reaction mixture was cooled to $\mathrm{rt}$, the mixture was extracted five times with $\mathrm{CH}_{2} \mathrm{Cl}_{2}$. The combined organic layers were washed twice with water. Then the organic layer was dried with anhydrous sodium sulphate and was concentrated under vacuum. Trituration of the residue in $\mathrm{Et}_{2} \mathrm{O}$ afforded the expected compound after filtration.

Yield 44\%. Yellow powder. Mp >260 ${ }^{\circ}$. ${ }^{1} \mathrm{H}$ NMR $\left(200 \mathrm{MHz}, \mathrm{DMSO}-d_{6}\right) \delta=8.70(\mathrm{~d}, J=1.8$ Hz, 1H, H-6), 8.50-8.45 (m, 2H, H-1 and H-9), 8.22 (dd, $J=1.6 ; 8.7$ Hz, 1H, H-8), 7.36 (d, $J$ $=3.8 \mathrm{~Hz}, 1 \mathrm{H}, \mathrm{H}-3), 7.18-7.05(\mathrm{~m}, 1 \mathrm{H}, \mathrm{H}-2) .{ }^{13} \mathrm{C} \mathrm{NMR}\left(50 \mathrm{MHz}, \mathrm{DMSO}-d_{6}\right) \delta=166.3,149.8$, 132.0, 131.7, 130.6, 130.1, 128.3, 119.4, 118.3, 115.5, 115.2, 110.8, 95.8. Anal. Calcd for $\mathrm{C}_{13} \mathrm{H}_{7} \mathrm{Cl}_{3} \mathrm{~N}_{2} \mathrm{O}_{2}: \mathrm{C}, 47.38 ; \mathrm{H}, 2.14 ; \mathrm{N}, 8.50$. Found: $\mathrm{C}, 47.52 ; \mathrm{H}, 2.21 ; \mathrm{N}, 8.47$.

\subsubsection{0. $N^{\prime}$-Hydroxy-4-(trichloromethyl)pyrrolo[1,2-a]quinoxaline-7- carboximidamide (10k)}

A 50-mL round-bottomed flask equipped with a condenser and a magnetic stir bar was charged with 4-(trichloromethyl)pyrrolo[1,2-a]quinoxaline-7-carbonitrile (311 mg, 1.0 mmol), hydroxylamine hydrochloride $(70 \mathrm{mg}, 1.0 \mathrm{mmol})$ and $5 \mathrm{~mL}$ of a mixture of $\mathrm{EtOH} / \mathrm{H}_{2} \mathrm{O}$ 7/3. The mixture was refluxed until disappearance of starting material (ca. $24 \mathrm{~h}$ ). After the reaction mixture was cooled to $\mathrm{rt}$ and the volatiles were removed, the mixture was extracted five times with $\mathrm{CH}_{2} \mathrm{Cl}_{2}$. The combined organic layers were washed twice with water. Then, the organic layer was dried with anhydrous sodium sulphate and was concentrated under vacuum. Purification by chromatography on silica gel (Petroleum Ether/EtOAc 7/3 then 5/5) gave the expected product.

Yield 74\%. Yellow powder. Mp $114^{\circ} \mathrm{C} .{ }^{1} \mathrm{H}$ NMR $\left(200 \mathrm{MHz}, \mathrm{DMSO}-d_{6}\right) \delta=9.92(\mathrm{~s}, 1 \mathrm{H}$, OH), 8.68 (m, $1 \mathrm{H}, \mathrm{H}-6), 8.42$ (d, $J=8.8 \mathrm{~Hz}, 1 \mathrm{H}, \mathrm{H}-9), 8.35$ (d, $J=1.6 \mathrm{~Hz}, 1 \mathrm{H}, \mathrm{H}-1), 8.10$ $(\mathrm{dd}, J=1.6 ; 8.8 \mathrm{~Hz}, 1 \mathrm{H}, \mathrm{H}-8), 7.36(\mathrm{dd}, J=1.0 ; 4.2 \mathrm{~Hz}, 1 \mathrm{H}, \mathrm{H}-3), 7.12$ (dd, $J=2.7 ; 4.2 \mathrm{~Hz}$, $1 \mathrm{H}, \mathrm{H}-2), 6.23$ (sl, 2H). ${ }^{13} \mathrm{C}$ NMR (50 MHz, DMSO- $\left.d_{6}\right) \delta=151.3,149.6,132.2,129.4,128.0$, 127.8, 127.6, 119.3, 117.9, 115.1, 114.9, 110.4, 96.0. HRMS (ESI): $\mathrm{m} / \mathrm{z}[\mathrm{M}+\mathrm{H}]^{+}$calcd for $\left[\mathrm{C}_{13} \mathrm{H}_{10} \mathrm{~N}_{4} \mathrm{OCl}_{3}\right]^{+}: 342.9915$; found: 342.9920 .

\subsubsection{Representative procedure for the preparation of 4-methyl-7-substituted-} pyrrolo[1,2-a]quinoxaline (11f,h) 
A small reactor equipped with a magnetic stir bar was charged with the appropriate 7substituted 4-(trichloromethyl)pyrrolo[1,2-a]quinoxaline $(1.1 \mathrm{mmol})$ and iron fine powder (880 mg, $15.8 \mathrm{mmol}$ ) in $15 \mathrm{~mL}$ of glacial $\mathrm{AcOH}$. The mixture was heated at reflux for $1 \mathrm{~h}$. After cooling at rt, the mixture was filtered through a pad of Celite ${ }^{\circledR}$ and thoroughly washed with $\mathrm{CHCl}_{3}$. Volatiles were removed under vaccum then the mixture was made alkaline with a saturated solution of $\mathrm{Na}_{2} \mathrm{CO}_{3}$. The aqueous layer was extracted with $\mathrm{CHCl}_{3}$, and then the organic layer was dried with anhydrous sodium sulphate and was concentrated under vacuum. Purification by chromatography on silica gel $\left(\mathrm{CHCl}_{3} /\right.$ acetone $)$ gave the expected product.

\subsubsection{4,7-Dimethylpyrrolo[1,2-a]quinoxaline (11f)}

Yield 56\%. Beige powder. $\mathrm{Mp} 140^{\circ} \mathrm{C} .{ }^{1} \mathrm{H} \mathrm{NMR}\left(200 \mathrm{MHz}, \mathrm{CDCl}_{3}\right) \delta=7.85(\mathrm{dd}, J=1.3,2.6$ Hz, 1H, H-1), 7.72 (s, 1H, H-6), 7.69 (d, $J=8.4$ Hz, 1H, H-9), 7.27 (dd, $J=2.2,7.6$ Hz, 1H, $\mathrm{H}-8), 6.90-6.80$ (m, 2H, H-2 and H-3), $2.73\left(\mathrm{~s}, 3 \mathrm{H}, \mathrm{CH}_{3}\right), 2.47\left(\mathrm{~s}, 3 \mathrm{H}, \mathrm{CH}_{3}\right) .{ }^{13} \mathrm{C} \mathrm{NMR}(50$ $\left.\mathrm{MHz}, \mathrm{CDCl}_{3}\right) \delta=153.5,135.3,135.2,128.8,128.3,126.1,125.2,114.5,113.6,113.5,106.9$, 21.8, 21.2. Anal. Calcd for $\mathrm{C}_{13} \mathrm{H}_{12} \mathrm{~N}_{2}$ : C, 79.56; H, 6.16; N, 14.27. Found: C, 79.89; H, 2.18; N, 13.99 .

\subsubsection{4-Methylpyrrolo[1,2-a]quinoxaline-7-carbonitrile (11h)}

Yield 57\%. White powder. Mp $140^{\circ} \mathrm{C} .{ }^{1} \mathrm{H}$ NMR $\left(200 \mathrm{MHz}, \mathrm{CDCl}_{3}\right) \delta=8.17(\mathrm{~d}, J=1.6 \mathrm{~Hz}$, 1H, H-1), 7.97-7.77 (m, 2H, H-6 and H-9), 7.68 (dd, $J=1.7 ; 8.5$ Hz, 1H, H-8), 7.01-6.87 (m, $2 \mathrm{H}, \mathrm{H}-2$ and $\mathrm{H}-3), 2.73$ (s, 3H, $\left.\mathrm{CH}_{3}\right) .{ }^{13} \mathrm{C} \mathrm{NMR}\left(50 \mathrm{MHz}, \mathrm{CDCl}_{3}\right) \delta=155.9,135.9,133.8$, 130.3, 129.8, 126.4 118.6, 115.4, 115.2, 114.9, 108.6, 108.4, 22.1. HRMS (ESI): $m / z$ [M + $\mathrm{H}]^{+}$calcd for $\left[\mathrm{C}_{13} \mathrm{H}_{10} \mathrm{~N}_{3}\right]^{+}:$208.0869; found: 208.0871 .

\subsection{Biology}

\subsubsection{In vitro Antiplasmodial evaluation}

In this study, a $\mathrm{K} 1$ culture-adapted $P$. falciparum strain resistant to chloroquine, pyrimethamine and proguanil was used in an in vitro culture. Maintenance in continuous culture was done as described previously by Trager and Jensen [38]. Cultures were maintained in fresh A+ human erythrocytes at $2.5 \%$ hematocrit in complete medium (RPMI 1640 with $25 \mathrm{mM}$ HEPES, $25 \mathrm{mM} \mathrm{NaHCO}, 10 \%$ of $\mathrm{A}+$ human serum) at $37{ }^{\circ} \mathrm{C}$ under reduced $\mathrm{O}_{2}$ atmosphere (gas mixture $14 \% \mathrm{O}_{2}, 6 \% \mathrm{CO}_{2}$, and $80 \% \mathrm{~N}_{2}$ ). Parasitaemia was maintained daily between $1 \%$ and $6 \%$. The P. falciparum drug susceptibility test was carried out by comparing quantities of DNA in treated and control cultures of parasite in human 
erythrocytes according to a SYBR Green I fluorescence-based method [39] using a 96-well fluorescence plate reader. Compounds, previously dissolved in DMSO (final concentration less than $0.5 \% \mathrm{v} / \mathrm{v}$ ) were incubated in a total assay volume of $200 \mu \mathrm{L}$ (RPMI, $4 \%$ hematocrit and $1 \%$ parasitaemia) for $72 \mathrm{~h}$ in a humidified atmosphere $\left(14 \% \mathrm{O}_{2}\right.$ and $\left.6 \% \mathrm{CO}_{2}\right)$ at $37{ }^{\circ} \mathrm{C}$, in 96-well flat bottom plates. Duplicate assays were performed for each sample. After incubation, $125 \mu \mathrm{L}$ supernatant was discarded and cells were washed twice with $125 \mu \mathrm{L} 1 \mathrm{X}$ PBS. $15 \mu \mathrm{L}$ re-suspended cells were transferred to 96-well flat bottom nonsterile black plates (Greiner Bio-one) already containing $15 \mu \mathrm{L}$ of the SYBR Green I lysis buffer (2X SYBR Green I, $20 \mathrm{mM}$ Tris base $\mathrm{pH}$ 7.5, $20 \mathrm{mM}$ EDTA, 0.008\% w/v saponin, 0.08\% w/v Triton X100). Negative control, treated by solvents (DMSO or $\mathrm{H}_{2} \mathrm{O}$ ) and positive controls (chloroquine and doxycycline) were added to each set of experiments. Plates were incubated for $15 \mathrm{~min}$ at $37{ }^{\circ} \mathrm{C}$ and then read on a TECAN Infinite F-200 spectrophotometer with excitation and emission wavelengths at 485 and $535 \mathrm{~nm}$, respectively. The concentrations of compounds required to induce a $50 \%$ decrease of parasite growth $\left(\mathrm{IC}_{50} \mathrm{~K} 1\right)$ were calculated from three independent experiments.

\subsubsection{In vitroCytotoxicity evaluation}

HepG2 cell line was maintained at $37{ }^{\circ} \mathrm{C}, 6 \% \mathrm{CO}_{2}, 14 \% \mathrm{O}_{2}, 80 \% \mathrm{~N}_{2}$, with $90 \%$ humidity in RPMI supplemented with 10\% fotal bovine serum, 1\% L-glutamine (200 mM) and penicillin (100 U/mL) / streptomycin $(100 \mu \mathrm{g} / \mathrm{mL})$ (complete RPMI medium). The evaluation of the tested molecules cytotoxicity on the HepG2 (hepatocarcinoma cell line purchased from ATCC, ref HB-8065) cell line was performed according to the method of Mosmann [40] with slight modifications. Briefly, $5.10^{3}$ cells in $100 \mu \mathrm{L}$ of complete medium were inoculated into each well of 96-well plates and incubated at $37{ }^{\circ} \mathrm{C}$ in a humidified $6 \% \mathrm{CO}_{2}$. After $24 \mathrm{~h}$ incubation, $100 \mu \mathrm{L}$ of medium with various product concentrations dissolved in DMSO (final concentration less than $0.5 \% \mathrm{v} / \mathrm{v}$ ) were added and the plates were incubated for $72 \mathrm{~h}$ at $37{ }^{\circ} \mathrm{C}$. Triplicate assays were performed for each sample. Each plate-well was then microscopeexamined for detecting possible precipitate formation before the medium was aspirated from the wells. $100 \mu \mathrm{L}$ of MTT (3-(4,5-dimethyl-2-thiazolyl)-2,5-diphenyl-2H-tetrazolium bromide) solution $(0.5 \mathrm{mg} / \mathrm{mL}$ in medium without FCS $)$ were then added to each well. Cells were incubated for $2 \mathrm{~h}$ at $37^{\circ} \mathrm{C}$. After this time, the MTT solution was removed and DMSO $(100 \mu \mathrm{L})$ was added to dissolve the resulting blue formazan crystals. Plates were shaken vigorously (700 rpm) for $10 \mathrm{~min}$. The absorbance was measured at $570 \mathrm{~nm}$ with $630 \mathrm{~nm}$ as reference wavelength using a BIO-TEK ELx808 Absorbance Microplate Reader. DMSO was 
used as blank and doxorubicin (purchased from Sigma Aldrich) as positive control. Cell viability was calculated as percentage of control (cells incubated without compound). The $50 \%$ cytotoxic concentration $\left(\mathrm{CC}_{50}\right)$ was determined from the dose-response curve by using the TableCurve software $2 \mathrm{D}$ v.5.0. $\mathrm{CC}_{50}$ values represent the mean value calculated from three independent experiments.

\section{Acknowledgement}

This work was supported by the CNRS and Aix-Marseille Université. The authors warmly thank Dr Vincent Remusat and Dr Rémi Legay for the ${ }^{1} \mathrm{H}$ - and ${ }^{13} \mathrm{C}$-NMR spectra recording.

\section{References}

[1] World Health Organization (WHO). World Malaria Report 2013. Geneva, Switzerland: World Health Organization (WHO).

[2] A.M. Dondorp, F. Nosten, P. Yi, D. Das, A.P. Phyo, J. Tarning, K.M. Lwin, F. Ariey, W. Hanpithakpong, S.J. Lee, P. Ringwald, K. Silamut, M. Imwong, K. Chotivanich, P. Lim, T. Herdman, S.S. An, S. Yeung, P. Singhasivanon, N.P.J. Day, N. Lindegardh, D. Socheat, N.J. White, Artemisinin Resistance in Plasmodium falciparum Malaria, N. Engl. J. Med. 361 (2009) 455-467.

[3] D.A. Fidock, Drug discovery: Priming the antimalarial pipeline, Nature. 465 (2010) 297298.

[4] S. Yeung, D. Socheat, V.S. Moorthy, A.J. Mills, Artemisinin resistance on the ThaiCambodian border, Lancet. 374 (2009) 1418-1419.

[5] O. Müller, A. Sié, P. Meissner, R.H. Schirmer, B. Kouyaté, Artemisinin resistance on the Thai-Cambodian border, Lancet. 374 (2009) 1419.

[6] C.H. Sibley, R.N. Price, Monitoring antimalarial drug resistance: Applying lessons learned from the past in a fast-moving present, Int. J. Parasitol. Drugs Drug Resist. 2 (2012) 126-133.

[7] M.P. Anthony, J.N. Burrows, S. Duparc, J.J. Moehrle, T.N.C. Wells, The global pipeline of new medicines for the control and elimination of malaria, Malar. J. 11 (2012) 316. 
[8] M.A. Biamonte, J. Wanner, K.G. Le Roch, Recent advances in malaria drug discovery, Bioorg. Med. Chem. Lett. 23 (2013) 2829-2843.

[9] V.M. Zhang, M. Chavchich, N.C. Waters, Targeting protein kinases in the malaria parasite: update of an antimalarial drug target, Curr. Top. Med. Chem. 12 (2012) 456-472.

[10] F.C. Schrader, M. Barho, I. Steiner, R. Ortmann, M. Schlitzer, The antimalarial pipeline - An update, Int. J. Med. Microbiol. 302 (2012) 165-171.

[11] Y. Kabri, N. Azas, A. Dumètre, S. Hutter, M. Laget, P. Verhaeghe, A. Gellis, P. Vanelle, Original quinazoline derivatives displaying antiplasmodial properties, Eur. J. Med. Chem. 45 (2010) 616-622.

[12] M.D. Crozet, C. Botta, M. Gasquet, C. Curti, V. Rémusat, S. Hutter, O. Chapelle, N. Azas, M. De Méo, P. Vanelle, Lowering of 5-nitroimidazole's mutagenicity: towards optimal antiparasitic pharmacophore, Eur. J. Med. Chem. 44 (2009) 653-659.

[13] A. Cohen, P. Verhaeghe, M.D. Crozet, S. Hutter, P. Rathelot, P. Vanelle, N. Azas, Tandem synthesis and in vitro antiplasmodial evaluation of new naphtho[2,1- $d]$ thiazole derivatives, Eur. J. Med. Chem. 55 (2012) 315-324.

[14] A. Gellis, A. Dumetre, G. Lanzada, S. Hutter, E. Ollivier, P. Vanelle, N. Azas, Preparation and antiprotozoal evaluation of promising beta-carboline alkaloids, Biomed Pharmacother. 66 (2012) 339-347.

[15] C. Kieffer, P. Verhaeghe, N. Primas, C. Castera-Ducros, A. Gellis, R. Rosas, S. Rault, P. Rathelot, P. Vanelle. Sonogashira cross-coupling reaction in 4-chloro-2trichloromethylquinazoline series is possible despite a side dimerization reaction, Tetrahedron 69 (2013) 2987-2995.

[16] P. Verhaeghe, N. Azas, M. Gasquet, S. Hutter, C. Ducros, M. Laget, S. Rault, P. Rathelot, P. Vanelle, Synthesis and antiplasmodial activity of new 4-aryl-2trichloromethylquinazolines, Bioorg. Med. Chem. Lett. 18 (2008) 396-401. 
[17] P. Verhaeghe, N. Azas, S. Hutter, C. Castera-Ducros, M. Laget, A. Dumètre, M. Gasquet, J.-P. Reboul, S. Rault, P. Rathelot, P. Vanelle, Synthesis and in vitro antiplasmodial evaluation of 4-anilino-2-trichloromethylquinazolines, Bioorg. Med. Chem. 17 (2009) 43134322.

[18] C. Castera-Ducros, N. Azas, P. Verhaeghe, S. Hutter, P. Garrigue, A. Dumètre, L. Mbatchi, M. Laget, V. Remusat, F. Sifredi, S. Rault, P. Rathelot, P. Vanelle, Targeting the human malaria parasite Plasmodium falciparum: In vitro identification of a new antiplasmodial hit in 4-phenoxy-2-trichloromethylquinazoline series, Eur. J. Med. Chem. 46 (2011) 4184-4191.

[19] P. Verhaeghe, A. Dumètre, C. Castera-Ducros, S. Hutter, M. Laget, C. Fersing, M. Prieri, J. Yzombard, F. Sifredi, S. Rault, P. Rathelot, P. Vanelle, N. Azas, 4-Thiophenoxy-2trichloromethyquinazolines display in vitro selective antiplasmodial activity against the human malaria parasite Plasmodium falciparum, Bioorg. Med. Chem. Lett. 21 (2011) 60036006.

[20] N. Primas, P. Verhaeghe, A. Cohen, C. Kieffer, A. Dumètre, S. Hutter, S. Rault, P. Rathelot, N. Azas, P. Vanelle, A New Synthetic Route to Original Sulfonamide Derivatives in 2-Trichloromethylquinazoline Series: A Structure-Activity Relationship Study of Antiplasmodial Activity, Molecules. 17 (2012) 8105-8117.

[21] P. Verhaeghe, P. Rathelot, A. Gellis, S. Rault, P. Vanelle, Highly efficient microwave assisted $\alpha$-trichlorination reaction of $\alpha$-methylated nitrogen containing heterocycles, Tetrahedron. 62 (2006) 8173-8176.

[22] P. Verhaeghe, P. Rathelot, S. Rault, P. Vanelle, Convenient preparation of original vinylic chlorides with antiparasitic potential in quinoline series, Lett. Org. Chem. 3 (2006) 891-897.

[23] Q. Liu, Y. Zhao, H. Fu, C. Cheng, Copper-Catalyzed Sequential N-Arylation and Aerobic Oxidation: Synthesis of Quinazoline Derivatives, Synlett. 24 (2013) 2089-2094. 
[24] M. Since, O. Khoumeri, P. Verhaeghe, M. Maillard-Boyer, T. Terme, P. Vanelle, First SNAr reaction using TDAE-initiated carbanions in quinazoline series, Tetrahedron Lett. 52 (2011) 3810-3813.

[25]J. Broggi, T. Terme, P. Vanelle, Organic Electron Donors as Powerful Single-Electron Reducing Agents in Organic Synthesis, Angew. Chem. Int. Ed. Engl. 53 (2014) 384-413.

[26] P. Mencarelli, F. Stegel, Formation of a neutral covalent adduct in the nucleophilic aromatic substitution reaction involving a carbon leaving group, J. Org. Chem. 50 (1985) $5415-5417$.

[27] C. Delpivo, G. Micheletti, C. Boga, A Green Synthesis of Quinoxalines and 2,3Dihydropyrazines, Synthesis. 45 (2013) 1546-1552.

[28] J. Guillon, P. Grellier, M. Labaied, P. Sonnet, J.-M. Léger, R. Déprez-Poulain, I. ForfarBares, P. Dallemagne, N. Lemaître, F. Péhourcq, J. Rochette, C. Sergheraert, C. Jarry, Synthesis, Antimalarial Activity, and Molecular Modeling of New Pyrrolo[1,2a]quinoxalines, Bispyrrolo[1,2-a]quinoxalines, Bispyrido[3,2-e]pyrrolo[1,2-a]pyrazines, and Bispyrrolo[1,2-a]thieno[3,2-e]pyrazines, J. Med. Chem. 47 (2004) 1997-2009.

[29] J. Guillon, S. Moreau, E. Mouray, V. Sinou, I. Forfar, S.B. Fabre, V. Desplat, P. Millet, D. Parzy, C. Jarry, P. Grellier, New ferrocenic pyrrolo[1,2-a]quinoxaline derivatives: Synthesis, and in vitro antimalarial activity, Bioorg. Med. Chem. 16 (2008) 9133-9144.

[30] J. Guillon, E. Mouray, S. Moreau, C. Mullié, I. Forfar, V. Desplat, S. Belisle-Fabre, N. Pinaud, F. Ravanello, A. Le-Naour, J.-M. Léger, G. Gosmann, C. Jarry, G. Déléris, P. Sonnet, P. Grellier, New ferrocenic pyrrolo[1,2-a]quinoxaline derivatives: Synthesis, and in vitro antimalarial activity - Part II, Eur. J. Med. Chem. 46 (2011) 2310-2326.

[31] L. van Heerden, T.T. Cloete, J.W. Breytenbach, C. de Kock, P.J. Smith, J.C. Breytenbach, D.D. N'Da, Synthesis and in vitro antimalarial activity of a series of bisquinoline and bispyrrolo[1,2-a]quinoxaline compounds, Eur. J. Med. Chem. 55 (2012) $335-345$. 
[32] H. Prunier, S. Rault, J.C. Lancelot, M. Robba, P. Renard, P. Delagrange, B. Pfeiffer, D.H. Caignard, R. Misslin, B. Guardiola-Lemaitre, M. Hamon, Novel and selective partial agonists of $5-\mathrm{HT}_{3}$ receptors. 2. Synthesis and biological evaluation of piperazinopyridopyrrolopyrazines, piperazinopyrroloquinoxalines, and piperazinopyridopyrroloquinoxalines, J. Med. Chem. 40 (1997) 1808-1819.

[33] J. Zhu, H. Xie, Z. Chen, S. Li, Y. Wu, Synthesis of 6-trifluoromethylindolo[1,2$c$ ]quinazolines and related heterocycles using $N$-(2-iodophenyl)trifluoroacetimidoyl chlorides as starting material via C-H bond functionalization, Chem. Commun. 47 (2011) 1512-1514.

[34] J.-G. Kim, D.O. Jang, Trifluoroacetylation of amines with trifluoroacetic acid in the presence of trichloroacetonitrile and triphenylphosphine, Tetrahedron Lett. 51 (2010) 683 685.

[35] T. Kato, N. Katagiri, A. Wagai, Trichloromethylquinolines : Synthesis and Reaction with Trimethyl Phosphite, Chem. Pharm. Bull. 29 (1981) 1069-1075.

[36] J. Guillon, I. Forfar, M. Mamani-Matsuda, V. Desplat, M. Saliège, D. Thiolat, S. Massip, A. Tabourier, J.-M. Léger, B. Dufaure, G. Haumont, C. Jarry, D. Mossalayi, Synthesis, analytical behaviour and biological evaluation of new 4-substituted pyrrolo[1,2a]quinoxalines as antileishmanial agents, Bioorg. Med. Chem. 15 (2007) 194-210.

[37] G.W.H. Cheeseman, B. Tuck, The synthesis of pyrrolo[1,2-a]quinoxalines from $N$-(2acylaminophenyl)pyrroles, J. Chem. Soc. C. (1966) 852-855.

[38] W. Trager, J.B. Jensen, Human malaria parasites in continuous culture, Science. 193 (1976) 673-675.

[39] W.A. Guiguemde, A.A. Shelat, D. Bouck, S. Duffy, G.J. Crowther, P.H. Davis, D.C. Smithson, M. Connelly, J. Clark, F. Zhu, M.B. Jiménez-Díaz, M.S. Martinez, E.B. Wilson, A.K. Tripathi, J. Gut, E.R. Sharlow, I. Bathurst, F. El Mazouni, J.W. Fowble, I. Forquer, P.L. McGinley, S. Castro, I. Angulo-Barturen, S. Ferrer, P.J. Rosenthal, J.L. Derisi, D.J. Sullivan, J.S. Lazo, D.S. Roos, M.K. Riscoe, M.A. Phillips, P.K. Rathod, W.C. Van Voorhis, V.M. 
Avery, R.K. Guy, Chemical genetics of Plasmodium falciparum, Nature. 465 (2010) 311315.

[40] T. Mosmann, Rapid colorimetric assay for cellular growth and survival: application to proliferation and cytotoxicity assays, J. Immunol. Methods. 65 (1983) 55-63. 


\section{Figures captions:}

Figure 1.Structures of the heterocyclic compounds which were selected from our chemical library for the preliminary in vitro antiplasmodial screening

Figure 2.Structures of $\mathbf{5 a}$ and its 2-substituted analogs in quinoxaline series

Figure 3.Structures of quinoxaline $\mathbf{5 f}$ and its position isomer in 2-trichloromethylquinazoline series

Figure 4. Structures of some previously described antimalarial pyrrolo[1,2-a]quinoxalines A-F

Figure 5.Rational for the synthesis of new 4-trichloromethylpyrrolo[1,2-a]quinoxaline derivatives as potential antiplasmodial agents

\section{Figures:}

\section{Figure 1.}

\section{Figure 2.}


Figure 3.

Figure 4.

Figure 5. 


\section{Schemes:}

Scheme 1.Synthesis of 7-substituted-pyrrolo[1,2-a]quinoxaline series.

Reagents and conditions : (i) 2,5-DMTHF, $\mathrm{AcOH}$, reflux, $2 \mathrm{~h}$; (ii) $\mathrm{SnCl}_{2} \cdot 2 \mathrm{H}_{2} \mathrm{O}, \mathrm{EtOH}$, reflux, 3 h or $\mathrm{BiCl}_{3}, \mathrm{NaBH}_{4}, \mathrm{EtOH}$, overnight; (iii) $\mathrm{ClCOCCl}_{3}, \mathrm{NEt}_{3}$, dioxane, reflux, 4 h; (iv) $\mathrm{POCl}_{3}$, pyridine, reflux, 4 h; (v) conc. $\mathrm{HCl}$, reflux, $5 \mathrm{~h}$; (vi) $\mathrm{NH}_{2} \mathrm{OH} . \mathrm{HCl}, \mathrm{EtOH} / \mathrm{H}_{2} \mathrm{O}$, reflux, $24 \mathrm{~h}$; (vii) $\mathrm{Fe}, \mathrm{AcOH}$, reflux, $1 \mathrm{~h}$; (viii) $\mathrm{PPh}_{3}, \mathrm{Et}_{3} \mathrm{~N}$, TFA, $\mathrm{CCl}_{3} \mathrm{CN}, \mathrm{CH}_{3} \mathrm{CN}$, rt, overnight. 
Tables:

Table 1.In vitro antiplasmodial and cytotoxicity evaluation of compounds 1-5.

\begin{tabular}{ccccc}
\hline Entry & Molecule & $\begin{array}{c}\text { HepG2 CC } \\
(\boldsymbol{\mu M})\end{array}$ & $\begin{array}{c}\text { K1 } \\
\text { P.falciparum IC }_{\mathbf{5 0}}(\boldsymbol{\mu M})\end{array}$ & $\begin{array}{c}\text { Selectivity } \\
\text { Index }^{\mathbf{c}}\end{array}$ \\
\hline $\mathbf{1}$ & $\mathbf{1 a}$ & $>125^{\mathrm{d}}$ & 8.5 & $>14.7$ \\
$\mathbf{2}$ & $\mathbf{1 b}$ & $>500^{\mathrm{d}}$ & $>50^{\mathrm{d}}$ & - \\
$\mathbf{3}$ & $\mathbf{2 a}$ & 180 & 8 & 22.5 \\
$\mathbf{4}$ & $\mathbf{2 b}$ & 179 & $>50^{\mathrm{d}}$ & $<3.6$ \\
$\mathbf{5}$ & $\mathbf{3 a}$ & $>500^{\mathrm{d}}$ & $>50^{\mathrm{d}}$ & - \\
$\mathbf{6}$ & $\mathbf{4 a}$ & 61 & 17.6 & 3.5 \\
$\mathbf{7}$ & $\mathbf{4 b}$ & 350 & $>50^{\mathrm{d}}$ & $<7$ \\
$\mathbf{8}$ & $\mathbf{5 a}$ & 3.1 & 1.5 & 2.1 \\
$\mathbf{9}$ & $\mathbf{5 b}$ & $>500^{\mathrm{d}}$ & $>50^{\mathrm{d}}$ & - \\
$\mathbf{1 0}$ & $\mathbf{5 c}$ & 19.3 & 12.8 & 1.5 \\
$\mathbf{1 1}$ & $\mathbf{5 d}$ & $>500^{\mathrm{d}}$ & $>50^{\mathrm{d}}$ & - \\
$\mathbf{1 2}$ & $\mathbf{5 0}$ & $>50^{\mathrm{d}}$ & - \\
$\mathbf{1 3}$ & $\mathbf{5 e}$ & 3.5 & 0.2 & 17.5 \\
Doxorubicine $^{\mathrm{a}}$ & $\mathbf{5 f}$ & 30 & - & - \\
Chloroquine $^{\mathbf{b}}$ & 20 & 0.6 & 50 \\
Doxycycline $^{\mathbf{b}}$ & & 6.0 & 3.3 \\
\hline
\end{tabular}

${ }^{\mathrm{a}}$ Doxorubicine was used as a cytotoxic reference-drug; ${ }^{\mathrm{b}}$ Chloroquinine and doxycycline were used as antimalarial reference-drugs, ${ }^{\mathrm{c}}$ Selectivity indexes were calculated according to the formula : $\mathrm{SI}=\mathrm{HepG} 2 \mathrm{CC}_{50} / \mathrm{K} 1 \mathrm{IC}_{50} .{ }^{\mathrm{d}}$ No activity was observed at the highest concentration tested. 
Table 2.In vitro antiplasmodial and cytotoxicity evaluation of the 4-trichloromethylpyrrolo[1,2-a]quinoxaline series

\begin{tabular}{|c|c|c|c|c|c|c|}
\hline Entry & Molecule & $\mathbf{R}_{\mathbf{1}^{-}}$ & $\mathbf{R}_{2-}$ & $\begin{array}{c}\text { HepG2CC } \\
{ }_{0}(\mu M)\end{array}$ & $\begin{array}{c}\text { PfK1 } \\
\text { IC }_{50}(\mu M)\end{array}$ & $\mathbf{S I}^{\mathrm{c}}$ \\
\hline 1 & 10a & $-\mathrm{CCl}_{3}$ & $-\mathrm{H}$ & 41.2 & 1.5 & 27.5 \\
\hline 2 & $10 \mathrm{~b}$ & $-\mathrm{CCl}_{3}$ & $-F$ & $>15^{\mathrm{d} *}$ & 1.7 & $>8.8$ \\
\hline 3 & $10 \mathrm{c}$ & $-\mathrm{CCl}_{3}$ & $-\mathrm{Cl}$ & 9.8 & 1.6 & 6.1 \\
\hline 4 & 10d & $-\mathrm{CCl}_{3}$ & $-\mathrm{Br}$ & $\mathrm{nd}^{*}$ & $\mathrm{nd}^{*}$ & - \\
\hline 5 & $10 e$ & $-\mathrm{CCl}_{3}$ & $-\mathrm{I}$ & 6.6 & 1.2 & 5.5 \\
\hline 6 & $10 f$ & $-\mathrm{CCl}_{3}$ & $-\mathrm{CH}_{3}$ & 53 & 2.4 & 22.1 \\
\hline 7 & $10 \mathrm{~g}$ & $-\mathrm{CCl}_{3}$ & $-\mathrm{OCH}_{3}$ & 35 & 4.2 & 8.3 \\
\hline 8 & $10 \mathrm{~h}$ & $-\mathrm{CCl}_{3}$ & $-\mathrm{CN}$ & 17 & 1.5 & 11.3 \\
\hline 9 & $10 \mathrm{i}$ & $-\mathrm{CCl}_{3}$ & $-\mathrm{COOCH}_{3}$ & $\mathrm{nd}^{*}$ & $\mathrm{nd}^{*}$ & - \\
\hline 10 & $10 \mathbf{j}$ & $-\mathrm{CCl}_{3}$ & $-\mathrm{COOH}$ & 106 & 22 & $<10.6$ \\
\hline 11 & $10 \mathrm{k}$ & $-\mathrm{CCl}_{3}$ & $-\mathrm{C}\left(\mathrm{NH}_{2}\right)=\mathrm{NOH}$ & $>15^{\mathrm{d}_{*}}$ & 1.75 & $>8.6$ \\
\hline 12 & $11 a$ & $-\mathrm{CH}_{3}$ & $-\mathrm{H}$ & 120 & $>50^{\mathrm{d}}$ & $<2.4$ \\
\hline 13 & $11 f$ & $-\mathrm{CH}_{3}$ & $-\mathrm{CH}_{3}$ & 57 & $>50^{\mathrm{d}}$ & $<4.7$ \\
\hline 14 & $11 \mathrm{~h}$ & $-\mathrm{CH}_{3}$ & $-\mathrm{CN}$ & $>31.5^{\mathrm{d}}$ & $>50^{\mathrm{d}}$ & - \\
\hline 15 & $12 \mathbf{a}$ & $-\mathrm{CF}_{3}$ & $-\mathrm{H}$ & 160 & $>50^{\mathrm{d}}$ & $<3.2$ \\
\hline \multicolumn{4}{|c|}{ Doxorubicine $^{a}$} & 0.2 & - & - \\
\hline \multicolumn{4}{|c|}{ Chloroquine $^{\mathrm{b}}$} & 30 & 0.6 & 50 \\
\hline \multicolumn{4}{|c|}{ Doxycycline $^{b}$} & 20 & 6.0 & 3.3 \\
\hline
\end{tabular}

${ }^{\mathrm{a}}$ Doxorubicine was used as a cytotoxic reference-drug; ${ }^{\mathrm{b}}$ Chloroquinine and doxycycline were used as antimalarial reference-drugs; ${ }^{c}$ Selectivity index (SI) was calculated according to the formula: $\mathrm{SI}=\mathrm{CC}_{50} / \mathrm{IC}_{50}$; ${ }^{\mathrm{d}}$ No activity was observed at the highest concentration tested; $*$ Not determined (nd) because of lack of solubility in the culture media. 
Graphical Abstract: 


\section{Highlights:}

Antiplasmodial screening of $\mathrm{CCl}_{3}$-substituted-nitrogen containing heterocycles was made.

2-Trichloromethylquinoxaline scaffold was the most interesting but was too cytotoxic.

Original 4-trichloromethylpyrrolo[1,2-a]quinoxalines analogs were prepared.

3 Compounds displayed an $\mathrm{IC}_{50}<2.4 \mu \mathrm{M}$ in vitro on $\mathrm{K} 1$ P. falciparum.

Cytotoxicity was assessed in parallel on the human HepG2 cell line showing good selectivity. 